\title{
Topological classification of Morse-Smale diffeomorphisms without heteroclinic curves on 3-manifolds
}

\author{
Ch. Bonatti \\ V. Grines \\ F. Laudenbach \\ O. Pochinka *
}

\section{Contents}

1 Introduction and formulation of the result 2

2 Dynamics of diffeomorphisms in the class $G(M)$

3 Compatible foliations $\quad 7$

4 Proof of the classification theorem $\quad 12$

4.1 Necessity . . . . . . . . . . . . . . . . . . . . . . . . . . . . . . . . . . . . . . .

4.2 Sufficiency . . . . . . . . . . . . . . . . . . . 13

5 Topological background

\begin{abstract}
We show that, up to topological conjugation, the equivalence class of a Morse-Smale diffeomorphism without heteroclinic curves on a 3-manifold is completely defined by an embedding of two-dimensional stable and unstable heteroclinic laminations to a characteristic space.
\end{abstract}

Key words: Morse-Smale diffeomorphism, topological classification, heteroclinic lamination MSC: 37C05, 37C15, 37C29, 37D15.

${ }^{*}$ The dynamics and necessary and sufficient conditions of the topological conjugation of Morse-Smale diffeomorphisms without heteroclinic curves on 3-manifolds (Sections 2, 4) were investigated with the support of the Russian Science Foundation (project 17-11-01041). The construction of the compatible foliations and topological research (Sections 3, 5) partially supported by RFBR (project nos. 15-01-03687-a, 16-51-10005-Ko_a), BRP at the HSE (project 90) in 2017 and by ERC Geodycon. 


\section{Introduction and formulation of the result}

In 1937 A. Andronov and L. Pontryagin [2] introduced the notion of a rough system of differential equation given in a bounded part of the plane, that is a system which preserves its qualitative properties under parameters variation if the variation is small enough. They proved that the flows generated by such systems are exactly the flows having the following properties:

1. the set of fixed points and periodic orbits is finite and all its elements are hyperbolic;

2. there are no separatrices going from one saddle to itself or to another one;

3. all $\omega$ - and $\alpha$-limit sets are contained in the union of fixed points and periodic orbits (limit cycles).

The above description characterizes the rough flows on the two-dimensional sphere also. After A. Mayer [16] in 1939, a similar result holds true on the 2-torus for flows having a closed section and no equilibrium states. A. Andronov and L. Pontryagin have shown also in [2] that the set of the rough flows is dense in the space of $C^{1}$-flows ${ }^{1}$. In 1962 M. Peixoto proved ([22], [23]) that the properties 1-3 are necessary and sufficient for a flow on any orientable surface of genus greater than zero to be structurally stable. He proved the density for these flows as well. Direct generalization of the properties of rough flows on surfaces leads to the following class of dynamical systems continuous or discrete, that is, flows or diffeomorphisms (cascades).

Definition 1.1 A smooth dynamical system given on an $n$-dimensional manifold $(n \geq 1) M^{n}$ is called Morse-Smale if:

1. its non-wandering set consists of a finite number of fixed points and periodic orbits where each of them is hyperbolic;

2. the stable and unstable manifolds $W_{p}^{s}, W_{q}^{u}$ of any pair of non-wandering points $p$ and $q$ intersect transversely.

Let $M$ be a given closed 3-dimensional manifold and $f: M \rightarrow M$ be a Morse-Smale diffeomorphism.

For $q=0,1,2,3$ denote by $\Omega_{q}$ the set of all periodic points of $f$ with $q$-dimensional unstable manifold. Let $\Omega_{f}$ be the union of all periodic points. Let us represent the dynamics of $f$ in the form "source-sink" in the following way. Set

$$
A_{f}=W_{\Omega_{0} \cup \Omega_{1}}^{u}, R_{f}=W_{\Omega_{2} \cup \Omega_{3}}^{s}, V_{f}=M \backslash\left(A_{f} \cup R_{f}\right) .
$$

We recall that a compact set $A \subset M$ is said to be an attractor of $f$ if there is a compact neighborhood $N$ of the set $A$ such that $f(N) \subset$ int $N$ and $A=\bigcap_{n \in \mathbb{N}} f^{n}(N)$; and $R \subset M$ is said to be a repeller of $f$ if it is an attractor of $f^{-1}$.

\footnotetext{
${ }^{1}$ This statement was not explicitly formulated in [2] and was mentioned for the first time in papers by E. Leontovich [15] and M. Peixoto [21]. G. Baggis [3] in 1955 made explicit some details of the proofs which were not completed in [2].
} 
By [11, Theorem 1.1] the set $A_{f}$ (resp. $R_{f}$ ) is an attractor (resp. a repeller) of $f$ whose topological dimension is equal to 0 or 1 . By [11, Theorem 1.2] the set $V_{f}$ is a connected 3manifold and $V_{f}=W_{A_{f} \cap \Omega_{f}}^{s} \backslash A_{f}=W_{R_{f} \cap \Omega_{f}}^{u} \backslash R_{f}$. Moreover, the quotient $\hat{V}_{f}=V_{f} / f$ is a closed connected 3-manifold and when $\hat{V}_{f}$ is orientable, then it is either irreducible or diffeomorphic to $\mathbb{S}^{2} \times \mathbb{S}^{1}$. The natural projection $p_{f}: V_{f} \rightarrow \hat{V}_{f}$ is an infinite cyclic covering. Therefore, there is a natural epimorphism from the the first homology group of $\hat{V}_{f}$ to $\mathbb{Z}$,

$$
\eta_{f}: H_{1}\left(\hat{V}_{f} ; \mathbb{Z}\right) \rightarrow \mathbb{Z}
$$

defined as follows: if $\gamma$ is a path in $V_{f}$ joining $x$ to $f^{n}(x), n \in \mathbb{Z}$, then $\eta_{f}$ maps the homology class of the cycle $p_{f} \circ \gamma$ to $n$.

The intersection with $V_{f}$ of the 2-dimensional stable manifolds of the saddle points of $f$ is an invariant 2-dimensional lamination $\Gamma_{f}^{s}$, with finitely many leaves, and which is closed in $V_{f}$. Each leaf of this lamination is obtained by removing from a stable manifold its set of intersection points with the 1-dimensional unstable manifold; this intersection is at most countable. As $\Gamma_{f}^{s}$ is invariant under $f$, it descends to the quotient in a compact 2-dimensional lamination $\hat{\Gamma}_{f}^{s}$ on $\hat{V}_{f}$. Note that each 2-dimensional stable manifold is a plane on which $f$ acts as a contraction, so that the quotient by $f$ of the punctured stable manifold is either a torus or a Klein bottle. Thus the leaves of $\hat{\Gamma}_{f}^{s}$ are either tori or Klein bottles which are punctured along at most countable set.

One defines in the same way the unstable lamination $\hat{\Gamma}_{f}^{u}$ as the quotient by $f$ of the intersection with $V_{f}$ of the 2-dimensional unstable manifolds. The laminations $\hat{\Gamma}_{f}^{s}$ and $\hat{\Gamma}_{f}^{u}$ are transverse.

Definition 1.2 The sets $\hat{\Gamma}_{f}^{s}$ and $\hat{\Gamma}_{f}^{u}$ are called the two-dimensional stable and unstable laminations associated with the diffeomorphism $f$.

A precise definition of what a lamination is will be given in Definition 2.1.

Definition 1.3 The collection $S_{f}=\left(\hat{V}_{f}, \eta_{f}, \hat{\Gamma}_{f}^{s}, \hat{\Gamma}_{f}^{u}\right)$ is called the scheme of the diffeomorphism $f$.

Definition 1.4 The schemes $S_{f}$ and $S_{f^{\prime}}$ of two Morse-Smale diffeomorphisms $f, f^{\prime}: M \rightarrow M$ are said to be equivalent if there is a homeomorphism $\hat{\varphi}: \hat{V}_{f} \rightarrow \hat{V}_{f^{\prime}}$ with following properties:

(1) $\eta_{f}=\eta_{f^{\prime}} \hat{\varphi}_{*}$;

(2) $\hat{\varphi}\left(\hat{\Gamma}_{f}^{s}\right)=\hat{\Gamma}_{f^{\prime}}^{s}$ and $\hat{\varphi}\left(\hat{\Gamma}_{f}^{u}\right)=\hat{\Gamma}_{f^{\prime}}^{u}$, meaning that $\hat{\varphi}$ maps leaf to leaf.

Using the above notion of a scheme in a series of papers by Ch. Bonatti, V. Grines, V. Medvedev, E. Pecou, O. Pochinka [5], [7], 8], [9], the problem of classification up to topological conjugacy of Morse-Smale diffeomorphisms on 3-manifolds has been solved in some particular cases. Recall that two diffeomorphisms $f$ and $f^{\prime}$ of $M$ are said to be topologically conjugate if there is a homeomorphism $h: M \rightarrow M$ which satisfies $f^{\prime} h=h f$.

In the present article, we give the topological classification of the Morse-Smale diffeomorphisms belonging to the subset $G(M)$ of the Morse-Smale diffeomorphisms $f: M \rightarrow M$ which have no heteroclinic curves (see Section 2). According to [6], when the ambient manifold is orientable, then it is either sphere $\mathbb{S}^{3}$ or the connected sum of a finite number copies of $\mathbb{S}^{2} \times \mathbb{S}^{1}$. 
Theorem 1 Two Morse-Smale diffeomorphisms in $G(M)$ are topologically conjugate if and only if their schemes are equivalent.

The structure of the paper is the following:

- In Section 2 we describe the dynamics of Morse-Smale diffeomorphisms and their space of wandering orbits.

- In Section 3 we construct a compatible system of neighborhoods, which is a key point for the construction of a conjugating homeomorphism.

- In Section 4 we construct a conjugating homeomorphism.

- Section 5 is an appendix of 3-dimensional topology. We prove there some topological lemmas which are used in Section 4.

\section{Dynamics of diffeomorphisms in the class $G(M)$}

In this section we introduce some notions connected with Morse-Smale diffeomorphisms on 3-manifold $M$. More detailed information on Morse-Smale diffeomorphisms is contained in [13. for example.

Let $f: M \rightarrow M$ be a Morse-Smale diffeomorphism. If $x$ is a periodic point its Morse index is the dimension of its unstable manifold $W_{x}^{u}$; the point $x$ is called a saddle point when its two invariant manifolds have positive dimension, that is, its Morse index is not extremal. A sink point has Morse index 0 and a source point has Morse index 3. The following notions are key concepts for describing how the stable manifolds of saddle points intersect the unstable ones. If $x, y$ are distinct saddle points of $f$ and $W_{x}^{u} \cap W_{y}^{s} \neq \emptyset$, then:

- if $\operatorname{dim} W_{x}^{s}<\operatorname{dim} W_{y}^{s}$, any connected component of $W_{x}^{u} \cap W_{y}^{s}$ is 1-dimensional and called a heteroclinic curve (see figure 1);

- if $\operatorname{dim} W_{x}^{s}=\operatorname{dim} W_{y}^{s}$, the set $W_{x}^{u} \cap W_{y}^{s}$ is countable; each of its points is called a heteroclinic point; the orbit of a heteroclinic point is called a heteroclinic orbit.

According to S. Smale [24], it is possible to define a partial order in the set of saddle points of a given Morse-Smale diffeomorphism $f$ as follows: for different periodic orbits $p \neq q$, one sets $p \prec q$ if and only if $W_{q}^{u} \cap W_{p}^{s} \neq \emptyset$. Smale proved that this relation is a partial order. In that case, it follows from [18, Lemma 1.5] that there is a sequence of different periodic orbits $p_{0}, \ldots, p_{n}$ satisfying the following conditions: $p_{0}=p, p_{n}=q$ and $p_{i} \prec p_{i+1}$. The sequence $p_{0}, \ldots, p_{n}$ is said to be an $n$-chain connecting $p$ to $q$. The length of the longest chain connecting $p$ to $q$ is denoted by $\operatorname{beh}(q \mid p)$. If $W_{q}^{u} \cap W_{p}^{s}=\emptyset$, we pose $b e h(q \mid p)=0$. For a subset $P$ of the periodic orbits let us set $b e h(q \mid P)=\max _{p \in P}\{b e h(q \mid p)\}$. The present paper is devoted to studying Morse-Smale diffeomorphisms in dimension 3 which have no heteroclinic curves. We recall from the introduction that this class of diffeomorphisms is denoted by $G(M)$. Let $f \in G(M)$. It follows from [11] that if the set $\Omega_{2}$ is empty then $R_{f}$ consists of a unique source. If $\Omega_{2} \neq \emptyset$, 


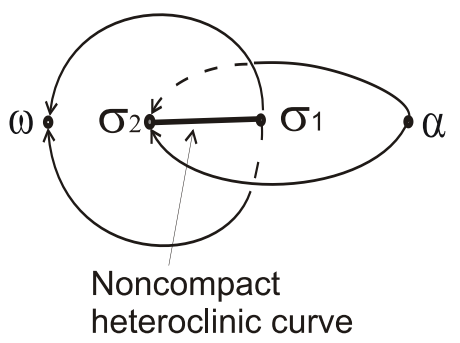

(a)

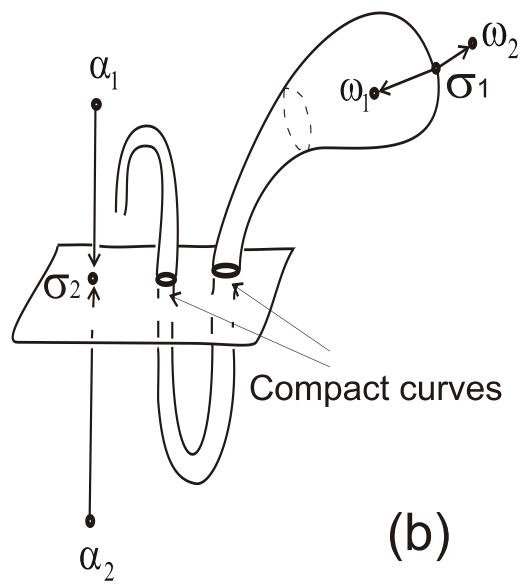

Figure 1: Heteroclinic curves

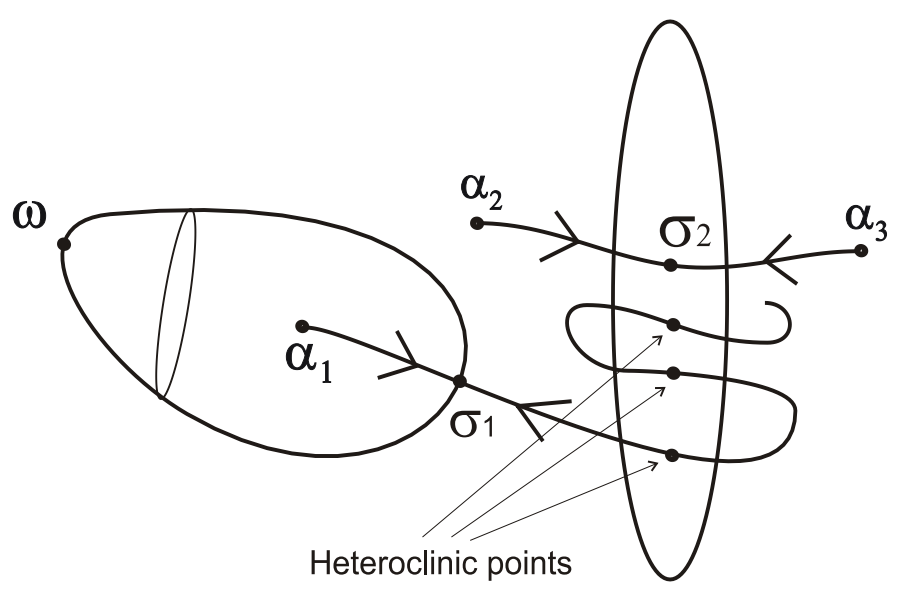

Figure 2: Heteroclinic points

denote by $n$ the length of the longest chain connecting two points of $\Omega_{2}$. Divide the set $\Omega_{2}$ into $f$-invariant disjoint parts $\Sigma_{0}, \Sigma_{1}, \ldots, \Sigma_{n}$ using the rule: $b e h\left(q \mid\left(\Omega_{2} \backslash q\right)\right)=0$ for each orbit $q \in \Sigma_{0}$ and $\operatorname{beh}\left(q \mid \Sigma_{i}\right)=1$ for each orbit $q \in \Sigma_{i+1}, i \in\{0, \ldots, n-1\}$. Since $\Omega_{1}$ for $f$ is $\Omega_{2}$ for $f^{-1}$, then it is possible to divide the periodic orbits of the set $\Omega_{1}$ into parts in a similar way. The absence of heteroclinic curves means that there are no chains connecting a saddle from $\Omega_{2}$ with a saddle from $\Omega_{1}$. Thus we explain all material for $\Omega_{2}$ and say that all is similar for $\Omega_{1}$.

Set $W_{i}^{u}:=W_{\Sigma_{i}}^{u}, W_{i}^{s}:=W_{\Sigma_{i}}^{s}$. Then, $R_{f}:=\bigcup_{i=0}^{n} c l\left(W_{i}^{s}\right)$, where $c l(\cdot)$ stands for the closure of $(\cdot)$. We now specify what a lamination is and which sort of regularity it may have. There are different possible notations. Here, we use the one which is given in [10, Definition 1.1.22].

Definition 2.1 Let $X$ be a n-dimensional and $Y \subset X$ be a closed subset. Let $q$ be an integer $0<q<n$. A codimension- $q$ lamination with support $Y$ is a decomposition $Y=\bigcup_{j \in J} L_{j}$ into pairwise disjoint smooth $(n-q)$-dimensional connected manifolds $L_{j}$, which are called the leaves. 
The family $L=\left\{L_{j}, j \in J\right\}$ is said to be a $C^{1,0}$-lamination ${ }^{2}$ if for every point $x \in Y$ the following conditions hold:

(1) There are an open neighborhood $U_{x} \subset X$ of $x$ and a homeomorphism $\psi: U_{x} \rightarrow \mathbb{R}^{n}$ such that $\psi$ maps every plaque, that is a connected component of $U_{x} \cap L_{j}$, into a codimension- $q$ subspace $\left\{\left(x^{1}, \ldots, x^{n}\right) \in \mathbb{R}^{n} \mid x^{n-q+1}=c^{n-q+1}, \ldots, x^{n}=c^{n}\right\}$. If $Y=X$ one says that $\mathcal{L}$ is foliation.

(2) The tangent plane field $T Y:=\bigcup_{j \in J} T L_{j}$ exists on $Y$ and is continuous.

By definition, two points belong to the same leaf of a lamination if they are linked by a path which is covered by finitely many plaques.

By abuse, a lamination and its support are generally denoted in the same way. We recall the $\lambda$-Lemma in the strong form which is proved in [19, Remarks p. 85].

Lemma 2.2 ( $\lambda$-lemma.) Let $f: X \rightarrow X$ be a diffeomorphism of an $n$-manifold, and let $p$ be a fixed point of $f$. Denote $W_{p}^{u}$ and $W_{p}^{s}$ be the unstable and stable manifold respectively; say $\operatorname{dim} W_{p}^{u}=m, 0<m<n$. Let $B^{s}$ be a compact subset of $W_{p}^{s}$ (containing $p$ or not) and let $F: B^{s} \rightarrow C^{1}\left(\mathbb{D}^{m}, X\right)$ be a continuous family of embedded closed $m$-disks of class $C^{1}$ transverse to $W_{p}^{s}$ and meeting $B^{s}$; set $F(x):=D_{x}^{u}$. Let $D^{u} \subset W_{p}^{u}$ be a compact $m$-disk and let $V \subset X$ be a compact $n$-ball such that $D^{u}$ is a connected component of $W_{p}^{u} \cap V$. Then, when $k$ goes to $+\infty$, the sequence $f^{k}\left(D_{x}^{u}\right) \cap V$ converges to $D^{u}$ in the $C^{1}$ topology uniformly for $x \in B^{s}$.

Notice that it is important for applications that $B^{s}$ may not contain the point $p$. Going back to our setting, a first application of the $\lambda$-lemma is that we have $W_{i}^{u} \subset \operatorname{cl}\left(W_{i+1}^{u}\right)$ and the closure of $\operatorname{cl}\left(W_{0}^{u}\right)=W_{0}^{u} \cup \Omega_{0}$. Moreover, $\operatorname{cl}\left(W_{n}^{u}\right) \cap\left(M \backslash \Omega_{0}\right)$ is a $C^{1,0}$-lamination of codimension one. From this one derives that $\hat{L}_{f}^{u}$ is also a $C^{1,0}$-lamination. Here is a typical example.

On Figure 3 there is a phase portrait of a diffeomorphism $f \in G(M)$ whose non-wandering set $\Omega_{f}$ consists of fixed points: one sink $\omega$, three saddle points $\Sigma_{0}=\sigma_{0}, \Sigma_{1}=\sigma_{1}, \Sigma_{2}=\sigma_{2}$ with two-dimensional unstable manifolds and four sources $\alpha_{0}, \alpha_{1}, \alpha_{2}, \alpha_{3}$. We will illustrate all further proofs with this diffeomorphism. For this case $V_{f}:=W_{\omega}^{s} \backslash\{\omega\}$. As the restriction of $f$ to the basin $W_{\omega}^{s}$ of $\omega$ is topologically conjugate to any homothety, $\hat{V}_{f}$ is diffeomorphic to $\mathbb{S}^{2} \times \mathbb{S}^{1}$. As $\left.f\right|_{W_{i}^{u}}$ is topologically conjugate to a homothety then $\left(W_{i}^{u} \backslash \Sigma_{i}\right) / f$ is diffeomorphic to the 2-torus; but this torus does not embed to $\hat{V}_{f}$, except when $i=0$. On Figure 4 there is the lamination associated with the diffeomorphism $f \in G(M)$ whose phase portrait is on Figure 3. On the left, the lamination is embedded in $\mathbb{S}^{2} \times \mathbb{S}^{1}$ which is seeen as the double of $\mathbb{S}^{2} \times \mathbb{D}^{1}$.

We are going to show that the topological classification of diffeomorphisms in the class $G(M)$ reduces to classifying some appropriate laminations $\hat{\Gamma}_{f}^{u}$ and $\hat{\Gamma}_{f}^{s}$. The technical key to the proof consists of constructing special foliations in some neighborhoods of the laminations.

\footnotetext{
${ }^{2}$ There are different possible notations. Here, we use the one which is given in [10, Definition 1.1.22].
} 


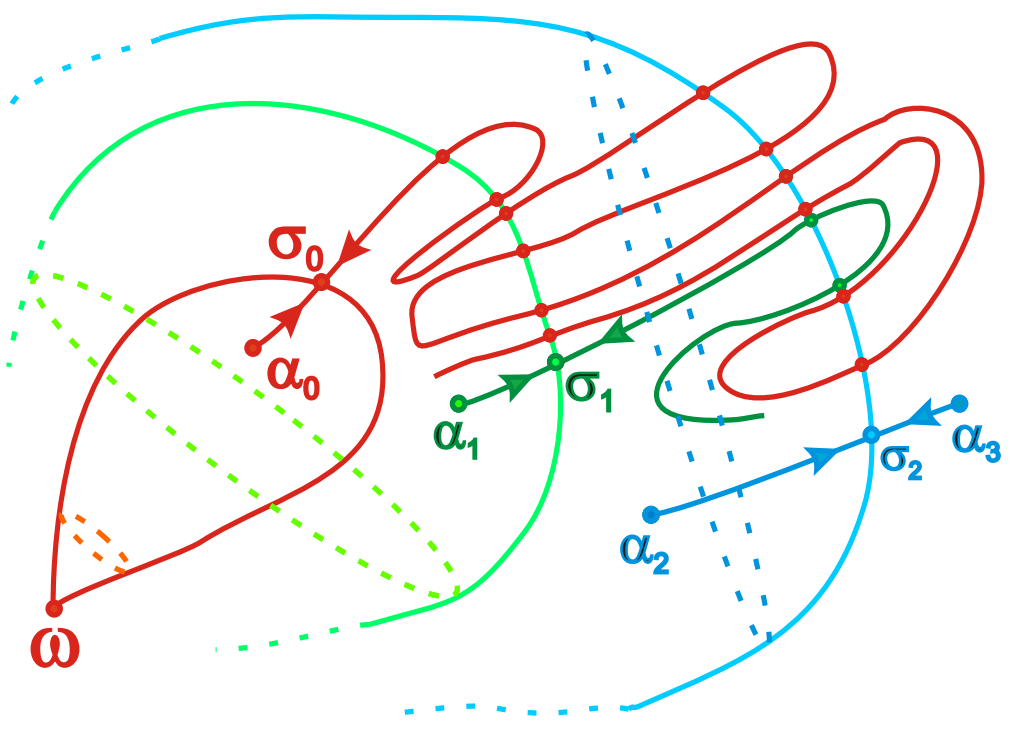

Figure 3: A phase portrait of a diffeomorphism from the class $G(M)$.

\section{Compatible foliations}

Let $f \in G(M)$. Recall that we divided the set $\Omega_{2}$ into the $f$-invariant parts $\Sigma_{0}, \ldots, \Sigma_{n}$. Using this partition, we explain how to construct compatible foliations (see Definition 3.3) around $W_{\Omega_{2}}^{u} \cup W_{\Omega_{2}}^{s}$. Similarly, it is possible to construct compatible foliations around $W_{\Omega_{1}}^{s} \cup W_{\Omega_{1}}^{u}$. In what follows, we give ourselves four models of concrete hyperbolic linear isomorphisms $\mathcal{E}_{\kappa, \nu} \in G L\left(\mathbb{R}^{3}\right), \kappa, \nu \in\{-,+\}$ given by the following formula:

$$
\mathcal{E}_{\kappa, \nu}\left(x_{1}, x_{2}, x_{3}\right)=\left(\kappa 2 x_{1}, 2 x_{2}, \nu \frac{x_{3}}{4}\right) .
$$

The origin $O$ is the unique fixed point which is a saddle point with unstable manifold $W_{O}^{u}=$ $O x_{1} x_{2}$ and stable manifold $W_{O}^{s}=O x_{3}$. If $\kappa=+($ resp. - ), the orientation of the unstable manifold is preserved (resp. reversed), and similarly for the orientation of the stable manifold with respect to $\nu$. We refer to each of them as the canonical diffeomorphism; it will be denoted by $\mathcal{E}$ ignoring the sign. For $p \in \Omega_{2}$, let $\operatorname{per}(p)$ denote the period of $f$ at $p$.

Definition 3.1 A neighborhood $N_{p}$ of a saddle point $p \in \Omega_{2}$ is called linearizable if there is a homeomorphism $\mu_{p}: N_{p} \rightarrow \mathcal{N}$ which conjugates the diffeomorphism $f^{\text {per }(p)}{ }_{N_{p}}$ to the canonical diffeomorphism $\left.\mathcal{E}\right|_{\mathcal{N}}$.

According to the local topological classification of hyperbolic fixed point [19, Theorem 5.5], every $p \in \Omega_{2}$ has a linearizable neighborhood $N_{p}$. For $t \in(0,1)$, set $\mathcal{N}^{t}:=\left\{\left(x_{1}, x_{2}, x_{3}\right) \in \mathbb{R}^{3} \mid\right.$ $\left.-t<\left(x_{1}^{2}+x_{2}^{2}\right) x_{3}<t\right\}$ and $\mathcal{N}:=\mathcal{N}^{1}$. The set $\mathcal{N}^{t}$ is invariant by the canonical diffeomorphism $\mathcal{E}$. By [24], $W_{p}^{s}$ and $W_{p}^{u}$ are smooth submanifolds of $M$. The boundary of $\mathcal{N}$ is the surface in $\mathbb{R}^{3}$ defined by the equations $\left(x_{1}^{2}+x_{2}^{2}\right) x_{3}= \pm 1$. The open manifold $N_{p}$ has a similar boundary 

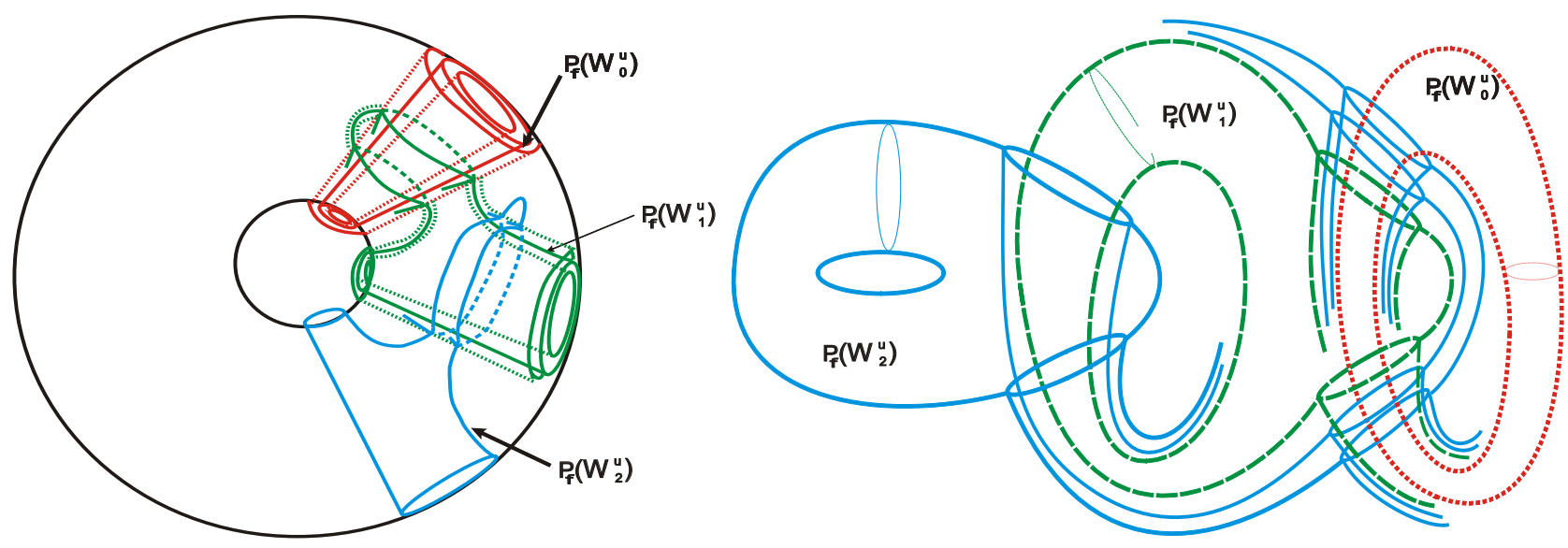

Figure 4: A lamination associated with the diffeomorphism $f \in G(M)$ whose phase portrait is pictured in Figure 3.

in $M$ denoted by $\partial N_{p}$. This boundary is formed by points which are not in $N_{p}$ but are limit points of arcs in $N_{p}$; it is distinct from its closure as a subset of $M$. Clearly, the linearizing homeomorphism $\mu_{p}$ extends to $\partial N_{p}$. For each $i \in\{0, \ldots, n\}$, choose some $p \in \Sigma_{i}$ and $\mu_{p}$ conjugating $f^{\text {per }(p)}$ to $\left.\mathcal{E}\right|_{\mathcal{N}}$. Then, for $k \in\{1, \ldots, \operatorname{per}(p)-1\}$ define $\mu_{f^{k}(p)}$ so that the next formula holds for every $x \in N_{f^{k-1}(p)}$ :

$$
\mu_{f^{k}(p)}(f(x))=\mu_{f^{k-1}(p)}(x) .
$$

We define a pair of transverse foliations $\left(\mathcal{F}^{u}, \mathcal{F}^{s}\right)$ in $\mathcal{N}$ in the following way:

- the leaves of $\mathcal{F}^{u}$ are the fibres in $\mathcal{N}$ of the projection $\left(x_{1}, x_{2}, x_{3}\right) \mapsto x_{3}$;

- the leaves of $\mathcal{F}^{s}$ are the fibres in $\mathcal{N}$ of the projection $\left(x_{1}, x_{2}, x_{3}\right) \mapsto\left(x_{1}, x_{2}\right)$.

By construction, $W_{O}^{u}$ and $W_{O}^{s}$ are leaves of $\mathcal{F}^{u}$ and $\mathcal{F}^{s}$ respectively. Let $N_{i}$ denote the union $\bigcup_{p \in \Sigma_{i}} N_{p}$. This is an $f$-invariant neighborhood of $\Sigma_{i}$. Let $\mu_{i}: N_{i} \rightarrow \mathcal{N}$ be the map whose restriction to $N_{p}$ is $\mu_{p}$. Thus, taking the pullback of them by $\mu_{i}$ gives a pair of $f$-invariant foliations $\left(F_{i}^{u}, F_{i}^{s}\right)$ on $N_{i}$ which are said to be linearizable. By construction, $W_{i}^{u}$ and $W_{i}^{s}$ are made of leaves of $F_{i}^{u}$ and $F_{i}^{s}$ respectively. Sometimes we want to deform the linearizable neighborhood $N_{p}$ by shrinking. Observe that the homotheties of ratio $\rho \in(0,1)$ act on $\mathcal{N}$ preserving $\mathcal{F}^{u}$ and $\mathcal{F}^{s}$ and map $\mathcal{N}$ to $\mathcal{N}^{\rho^{3}}$. By conjugation, similar contractions $c_{\rho}$ are available in $N_{p}$ for every $p \in \Omega_{2}$. The neighborhood $c_{\rho}\left(N_{p}\right)$ is said to be obtained from $N_{p}$ by shrinking.

Lemma 3.2 For every $\rho \in(0,1)$, the shrunk neighborhood $c_{\rho}\left(N_{p}\right)$ is linearizable. Generically, the boundary of $c_{\rho}\left(N_{p}\right)$ does not contain any heteroclinic point.

Proof: For a given $\mu_{p}$, we define $\mu_{p}^{\rho}$ as follows: its domain is $\mu_{p}^{-1}\left(\mathcal{N}^{\rho^{3}}\right)$ and, on this domain, it is defined by $\mu_{p}^{\rho}=c_{\rho}^{-1} \circ \mu_{p}$. Its range is $\mathcal{N}$. Since the heteroclinic points form a countable 
set, for almost every $\rho \in(0,1)$ the boundary of the domain of $\mu_{p}^{\rho}$ avoids the heteroclinic points.

Observe that the canonical diffeomorphism and the contraction $c_{\rho}$ keep both foliations invariant. Recall the $f$-invariant partition $\Omega_{2}=\Sigma_{0} \sqcup \Sigma_{1} \sqcup \ldots \sqcup \Sigma_{n}$. Let us introduce the following notations:

- for any $t \in(0,1)$, set $N_{p}^{t}:=\mu_{p}^{-1}\left(\mathcal{N}^{t}\right)$ and $N_{i}^{t}:=\bigcup_{p \in \Sigma_{i}} N_{p}^{t}$;

- for any point $x \in N_{i}$, denote $F_{i, x}^{u}\left(\operatorname{resp} . F_{i, x}^{s}\right)$ the leaf of the foliation $F_{i}^{u}\left(\right.$ resp. $\left.F_{i}^{s}\right)$ passing through $x$;

- for each point $x \in N_{i}$, set $x_{i}^{u}=W_{i}^{u} \cap F_{i, x}^{s}$ and $x_{i}^{s}=W_{i}^{s} \cap F_{i, x}^{u}$. Thus, we have $x=\left(x_{i}^{u}, x_{i}^{s}\right)$ in the coordinates defined by $\mu_{i}$.

We also introduce the radial functions $r_{i}^{u}, r_{i}^{s}: N_{i} \rightarrow[0,+\infty)$ defined by:

$$
r_{i}^{u}(x)=\left\|\mu_{i}\left(x_{i}^{u}\right)\right\|^{2} \quad \text { and } \quad r_{i}^{s}(x)=\left|\mu_{i}\left(x_{i}^{s}\right)\right| .
$$

With this definition at hand, the neighborhood $N_{i}^{t}$ ot $\Sigma_{i}$ is defined by the inequality

$$
r_{i}^{u}(x) r_{i}^{s}(x)<t
$$

Observe that the radial function $r_{i}^{s}$ endows each stable separatrix of $p \in \Sigma_{p}$ with a natural order which will be used later in the proof of Theorem 1 .

Definition 3.3 The linearizable neighborhoods $N_{0}, \ldots, N_{n}$ are called compatible if, for any $0 \leq i<j \leq n$ and $x \in N_{i} \cap N_{j}$, the following holds:

$$
F_{j, x}^{s} \cap N_{i} \subset F_{i, x}^{s} \text { and } F_{i, x}^{u} \cap N_{j} \subset F_{j, x}^{u} .
$$

If linearizable neighborhoods are compatible, they remain so after some of them are shrunk.

Remark 3.4 The notion of compatible foliations is a modification of the admissible systems of tubular families introduced by J. Palis and S. Smale in [18] and [20].

We introduce the following notation:

- For $i \in\{0, \ldots, n\}$, set $A_{i}:=A_{f} \cup \bigcup_{j=0}^{i} W_{j}^{u}$,

$V_{i}:=W_{A_{i} \cap \Omega_{f}}^{s} \backslash A_{i}, \quad \hat{V}_{i}:=V_{i} / f$. Observe that $f$ acts freely on $V_{i}$ and denote the natural projection by $p_{i}: V_{i} \rightarrow \hat{V}_{i}$.

- For $j, k \in\{0, \ldots, n\}$ and $t \in(0,1)$, set $\hat{W}_{j, k}^{s}=p_{k}\left(W_{j}^{s} \cap V_{k}\right), \hat{W}_{j, k}^{u}=p_{k}\left(W_{j}^{u} \cap V_{k}\right)$, $\hat{N}_{j, k}^{t}=p_{k}\left(N_{j}^{t} \cap V_{k}\right)$.

- $L^{u}:=\bigcup_{i=0}^{n} W_{i}^{u}, L^{s}:=\bigcup_{i=0}^{n} W_{i}^{s}, L_{i}^{u}:=L^{u} \cap V_{i}, L_{i}^{s}:=L^{s} \cap V_{i}, \hat{L}_{i}^{u}:=p_{i}\left(L_{i}^{u}\right), \hat{L}_{i}^{s}:=p_{i}\left(L_{i}^{s}\right)$. 
Theorem 2 For each diffeomorphism $f \in G(M)$ there exist compatible linearizable neighborhoods of all saddle points whose Morse index is 2.

Proof: The proof consists of three steps.

Step 1. Here, we prove the following claim.

Lemma 3.5 There exist $f$-invariant neighborhoods $U_{0}^{s}, \ldots, U_{n}^{s}$ of the sets $\Sigma_{0}, \ldots, \Sigma_{n}$ respectively, equipped with two-dimensional $f$-invariant foliations $F_{0}^{u}, \ldots, F_{n}^{u}$ of class $C^{1,0}$ such that the following properties hold for each $i \in\{0, \ldots, n\}$ :

(i) the unstable manifolds $W_{i}^{u}$ are leaves of the foliation $F_{i}^{u}$ and each leaf of the foliation $F_{i}^{u}$ is transverse to $L_{i}^{s}$;

(ii) for any $0 \leq i<k \leq n$ and $x \in U_{i}^{s} \cap U_{k}^{s}$, we have the inclusion $F_{k, x}^{u} \cap U_{i}^{s} \subset F_{i, x}^{u}$.

Proof: Let us prove this by a decreasing induction on $i$ from $i=n$ to $i=0$. For $i=n$, it follows from the definition of $V_{n}$ that $\left(W_{n}^{s} \backslash \Sigma_{n}\right) \subset V_{n}$. Since $f$ acts freely and properly on $W_{n}^{s}$, the quotient $\hat{W}_{n, n}^{s}$ is a smooth submanifold of $\hat{V}_{n}$; it consists of finitely many circles. The lamination $\hat{L}_{n}^{s}$ accumulates on $\hat{W}_{n, n}^{s}$. Choose an open tubular neighborhood $\hat{N}_{n}^{s}$ of $\hat{W}_{n, n}^{s}$ in $\hat{V}_{n}$; denote its projection by $\pi_{n}^{u}: \hat{N}_{n}^{s} \rightarrow \hat{W}_{n, n}^{s}$. Its fibers form a 2-disc foliation $\left\{d_{n, x}^{u} \mid x \in \hat{W}_{n, n}^{s}\right\}$ transverse to $\hat{W}_{n, n}^{s}$. Since $\hat{L}_{n}^{s}$ is a $C^{1,0}$-lamination containing $\hat{W}_{n, n}^{s}$, each plaque of $\hat{W}_{n, n}^{s}$ is the $C^{1}$-limit of any sequence of plaques approaching it $C^{0}$. Therefore, if the tube $\hat{N}_{n}^{s}$ is small enough, its fibers are transverse to $\hat{L}_{n}^{s}$.

Set $U_{n}^{s}:=p_{n}^{-1}\left(\hat{N}_{n}^{s}\right) \cup W_{n}^{u}$. This is an open set of $M$ which carries a foliation $F_{n}^{u}$ defined by taking the preimage of the fibers of $\pi_{n}^{u}$ and by adding $W_{n}^{u}$ as extra leaves. This is the requested foliation satisfying (i) and (ii) for $i=n$. Notice that the plaques of $F_{n}^{u}$ are smooth and by the $\lambda$-lemma, for any compact disc $B$ in $W_{n}^{u}$ there is $\varepsilon>0$ such that every plaque of $F_{n}^{u}$ which is $\varepsilon$-close to $B$ in topology $C^{0}$ is also $\varepsilon$-close to $B$ in topology $C^{1}$. Hence, $F_{n}^{u}$ is a $C^{1,0}$-foliation.

For the induction, we assume the construction is done for every $j>i$ and we have to construct an $f$-invariant neighborhood $U_{i}^{s}$ of the saddle points in $\Sigma_{i}$ carrying an $f$-invariant foliation $F_{i}^{u}$ satisfying (i) and (ii). Moreover, by genericity the boundary $\partial U_{j}^{s}, j>i$, is assumed to avoid all heteroclinic points. For $j>i$, let $\hat{U}_{j, i}^{s}:=p_{i}\left(U_{j}^{s} \cap V_{i}\right)$ and $\hat{F}_{j, i}^{u}:=p_{i}\left(F_{j}^{u} \cap V_{i}\right)$. For the same reason as in the case $i=n$, the set $\hat{W}_{i, i}^{s}$ is a smooth submanifold of $\hat{V}_{i}$ consisting of circles. Choose a tubular neighborhood $\hat{N}_{i}^{s}$ of $\hat{W}_{i, i}^{s}$ with a projection $\pi_{i}^{u}: \hat{N}_{i}^{s} \rightarrow \hat{W}_{i, i}^{s}$ whose fibers are 2-discs. Similarly, $\left(W_{i+1}^{u} \backslash \Sigma_{i}\right) \subset V_{i}$ and, hence, $\hat{W}_{i+1, i}^{u}$ is a compact submanifold, consisting of finitely many tori or Klein bottles. The set $\hat{L}_{i}^{u}$ is a compact lamination and its intersection with $\hat{W}_{i, i}^{s}$ consists of a countable set of points which are the projections of the heteroclinic points belonging to the stable manifolds $W_{i}^{s}$. Actually, there is a hierarchy in $\hat{L}_{i}^{u} \cap \hat{W}_{i, i}^{s}$ which we are going to describe in more details.

Set $H_{k}:=\hat{W}_{i+k, i}^{u} \cap \hat{W}_{i, i}^{s}$ for $k>0$. Since $\hat{W}_{i+1, i}^{u}$ is compact, $H_{1}$ is a finite set: $H_{1}=$ $\left\{h_{1}^{1}, \ldots, h_{t(1)}^{1}\right\}$. We are given neighborhoods, called boxes, $B_{\ell}^{1}, \ell=1, \ldots, t(1)$, about these points, namely, the connected components of $\hat{U}_{i+1, i}^{s} \cap \hat{N}_{i}^{s}$. Due to the fact that $\partial \hat{U}_{i+1, i}^{s}$ contains no heteroclinic point, $\partial \hat{U}_{i+1, i}^{s} \cap \hat{W}_{i, i}^{s}$ is isolated from $\hat{L}_{i}^{u}$. Therefore, if the tube $\hat{N}_{i}^{s}$ is small enough, 
$\hat{L}_{i}^{u}$ does not intersect $\partial \hat{U}_{i+1, i}^{s} \cap \hat{N}_{i}^{s}$. Then, by shrinking $U_{j}^{s}, j>i+1$ (in the sense of Lemma 3.2 if necessary, we may guarantee that $\hat{U}_{j, i}^{s} \cap \hat{N}_{i}^{s}$ is disjoint from $\partial \hat{U}_{i+1, i}^{s} \cap \hat{N}_{i}^{s}$.

Since $\hat{W}_{i+2, i}^{u}$ accumulates on $\hat{W}_{i+1, i}^{u}$, there are only finitely many points of $H_{2}$ outside of all boxes $B_{\ell}^{1}, \ell=1, \ldots, t(1)$. Let $\bar{H}_{2}:=\left\{h_{1}^{2}, \ldots, h_{t(2)}^{2}\right\}$ be this finite set. The open set $\hat{U}_{i+2, i}^{s}$ is a neighborhood of $\bar{H}_{2}$. The connected components of $\hat{U}_{i+2, i}^{s} \cap \hat{N}_{i}^{s}$ which contain points of $\bar{H}_{2}$ will be the box $B_{\ell}^{2}$ for $\ell=1, \ldots, t(2)$. We argue with $B_{\ell}^{2}$ with respect to $\hat{L}_{i}^{u}$ and the neighborhoods $\hat{U}_{j, i}, j>i+1$, in a similar manner as we do with $B_{\ell}^{1}$. And so on, until $\bar{H}_{n}$.

Due to the induction hypothesis, each above-mentioned box is foliated. Namely, $B_{\ell}^{1}$ is foliated by $\hat{F}_{i+1, i}^{u}$; the box $B_{\ell}^{2}$ is foliated by $\hat{F}_{i+2, i}^{u}$, and so on. But the leaves are not contained in fibres of $\hat{N}_{i}$; even more, not every leaf intersects $\hat{W}_{i, i}^{s}$. We have to correct this situation in order to construct the foliation $F_{i}^{u}$ satisfying the requested conditions (i) and (ii). For every $j>i$, the foliation $F_{j}^{u}$ may be extended to the boundary $\partial U_{j}^{s}$ and a bit beyond. Once this is done, if $\hat{N}_{i}^{s}$ is enough shrunk, each leaf of $\hat{F}_{i+k, i}^{u}$ through $x \in B_{\ell}^{k}$ intersects $\hat{W}_{i, i}^{s}$ (it is understood that the boxes are intersected with the shrunk tube without changing their names). Thus, we have a projection along the leaves $\pi_{k, \ell}: B_{\ell}^{k} \rightarrow \hat{W}_{i, i}^{s}$; but, the image of $\pi_{k, \ell}$ is larger than $B_{\ell}^{k} \cap \hat{W}_{i, i}^{s}$. Then, we choose a small enlargement $B_{\ell}^{\prime k}$ of $B_{\ell}^{k}$ such that $B_{\ell}^{\prime k} \backslash B_{\ell}^{k}$ is foliated by $\hat{F}_{i+k, i}^{u}$ and avoids the lamination $\hat{L}_{i}^{u}$. On $B_{\ell}^{\prime k} \backslash B_{\ell}^{k}$ we have two projections: one is $\hat{\pi}_{i}^{u}$ and the other one is $\pi_{k, \ell}$. We are going to interpolate between both using a partition of unity (we do it for $B_{\ell}^{k}$ but it is understood that it is done for all boxes). Let $\phi: \hat{N}_{i}^{s} \rightarrow[0,1]$ be a smooth function which equals 1 near $B_{\ell}^{k}$ and whose support is contained in $B_{\ell}^{\prime k}$. Define a global $C^{1}$ retraction $\hat{q}: \hat{N}_{i}^{s} \rightarrow \hat{W}_{i, i}^{s}$ by the formula

$$
\hat{q}(x)=(1-\phi(x)) \hat{\pi}_{i}^{u}(x)+\phi(x)\left(\pi_{k, \ell}(x)\right) .
$$

Here, we use an affine manifold structure on each component of $\hat{W}_{i, i}^{s}$ by identifying it with the 1 -torus $\mathbb{T}:=\mathbb{R} / \mathbb{Z}$. So, any positively weighted barycentric combination makes sense for a pair of points sufficiently close. When $x \in \hat{W}_{i, i}^{s}$, we have $\hat{q}(x)=x$. Then, by shrinking the tube $\hat{N}_{i}^{s}$ once more if necessary we make $\hat{q}$ be a fibration whose fibres are transverse to the lamination $\hat{L}_{i}^{s}$ and we make each leaf of $\hat{F}_{j, i}^{u}, j>i$, in every box $B_{k}^{\ell}$ be contained in a fibre of $q$. Henceforth, taking the preimage of that tube (and its fibration) by $p_{i}$ and adding the unstable manifold $W_{i}^{u}$ provide the requested $U_{i}^{s}$ and its foliation $F_{i}^{u}$ satisfying the required properties. Thus, the induction is proved.

We also have the following statement.

Lemma 3.6 There exist $f$-invariant neighborhoods $U_{0}^{u}, \ldots, U_{n}^{u}$ of the sets $\Sigma_{0}, \ldots, \Sigma_{n}$ respectively, equipped with one-dimensional $f$-invariant foliations $F_{0}^{s}, \ldots, F_{n}^{s}$ of class $C^{1,0}$ such that the following properties hold for each $i \in\{0, \ldots, n\}$ :

(iii) the stable manifold $W_{i}^{s}$ is a leaf of the foliation $F_{i}^{s}$ and each leaf of the foliation $F_{i}^{s}$ is transverse to $L_{i}^{u}$;

(iv) for any $0 \leq j<i$ and $x \in U_{i}^{u} \cap U_{j}^{u}$, we have the inclusion $\left(F_{j, x}^{s} \cap U_{i}^{u}\right) \subset F_{i, x}^{s}$.

Proof: The proof is done by an increasing induction from $i=0$; it is skipped due to similarity to the previous one. 
Before entering Step 2, we recall the definition of fundamental domain for a free action.

Definition 3.7 Let $g: X \rightarrow X$ be a homeomorphism acting freely on $X$. A closed subset $D \subset X$ is said to be a fundamental domain for the action of $g$ if the following properties hold:

1. $D$ is the closure of its interior $\stackrel{\circ}{D}$;

2. $g^{k}(\stackrel{\circ}{D}) \cap D=\emptyset$ for every integer $k \neq 0$;

3. $X$ is the union $\cup_{k \in \mathbb{Z}} g^{k}(D)$.

Step 2. We prove the following statement for each $i=0, \ldots, n$.

Lemma 3.8 (v) There exists an $f$-invariant neighborhood $\tilde{N}_{i}$ of the set $\Sigma_{i}$ contained in $U_{i}^{s} \cap U_{i}^{u}$ and such that the restrictions of the foliations $F_{i}^{u}$ and $F_{i}^{s}$ to $\tilde{N}_{i}$ are transverse.

Proof: For this aim, let us choose a fundamental domain $K_{i}^{s}$ of the restriction of $f$ to $W_{i}^{s} \backslash \Sigma_{i}$ and take a tubular neighborhood $N\left(K_{i}^{s}\right)$ of $K_{i}^{s}$ whose disc fibres are contained in leaves of $F_{i}^{u}$. By construction, $F_{i}^{u}$ is transverse to $W_{i}^{s}$ and, according to the Lemma 3.6, $F_{i}^{s}$ is a $C^{1,0}$-foliation. Therefore, if the tube $N\left(K_{i}^{s}\right)$ is small enough, $F_{i}^{u}$ is transverse to $F_{i}^{s}$ in $N\left(K_{i}^{s}\right)$. Set

$$
\tilde{N}_{i}:=W_{i}^{u} \bigcup_{k \in \mathbb{Z}} f^{k}\left(N\left(K_{i}^{s}\right)\right)
$$

This is a neighborhood of $\Sigma_{i}$; it satisfies condition (v) and the previous properties (i)-(iv) still hold. A priori the boundary of $\tilde{N}_{i}$ is only piecewise smooth; but, by choosing $N\left(K_{i}^{s}\right)$ correctly at its corners we may arrange that $\partial \tilde{N}_{i}$ be smooth.

Step 3. For proving Theorem 2 it remains to show the existence of linearizable neighborhoods $N_{i} \subset \tilde{N}_{i}, i=0, \ldots, n$, for which the required foliations are the restriction to $N_{i}$ of the foliations $F_{i}^{u}$ and $F_{i}^{s}$. For each orbit of $f$ in $\Sigma_{i}$, choose one $p$. Let $\tilde{N}_{p}$ be a connected component of $\tilde{N}_{i}$ containing $p$. There is a homeomorphism $\varphi_{p}^{u}: W_{p}^{u} \rightarrow W_{O}^{u}$ (resp. $\left.\varphi_{p}^{s}: W_{p}^{s} \rightarrow W_{O}^{s}\right)$ conjugating the diffeomorphisms $\left.f^{p e r(p)}\right|_{W_{p}^{u}}$ and $\left.\mathcal{E}\right|_{W_{O}^{u}}$ (resp. $\left.f^{p e r(p)}\right|_{W_{p}^{s}}$ and $\left.\left.\mathcal{E}\right|_{W_{O}^{s}}\right)$. In addition, for any point $z \in \tilde{N}_{p}$ there is unique pair of points $z_{s} \in W_{p}^{s}, z_{u} \in W_{p}^{u}$ such that $z=F_{i, z_{u}}^{s} \cap F_{i, z_{s}}^{u}$. We define a topological embedding $\tilde{\mu}_{p}: \tilde{N}_{p} \rightarrow \mathbb{R}^{3}$ by the formula $\tilde{\mu}_{p}(z)=\left(x_{1}, x_{2}, x_{3}\right)$ where $\left(x_{1}, x_{2}\right)=\varphi_{p}^{u}\left(z_{u}\right)$ and $x_{3}=\varphi_{p}^{s}\left(z_{s}\right)$. Since the foliations $F_{i}^{u}$ and $F_{i}^{s}$ are $f$-invariant, this definition makes $\tilde{\mu}_{p}$ conjugate the restriction $\left.f^{p e r(p)}\right|_{\tilde{N}^{p}}$ to $\mathcal{E}^{p e r(p)}$. For $k=1, \ldots, \operatorname{per}(p)-1$, set $\tilde{N}_{f^{k}(p)}:=f^{k}\left(\tilde{N}_{p}\right)$ and define $\tilde{\mu}_{f^{k}(p)}$ so that the equivariance formula holds: $\tilde{\mu}_{f^{k}(p)}\left(f^{k}(x)\right)=a^{k} \tilde{\mu}_{p}(x)$ for every $x \in \tilde{N}_{p}$. Choose $t_{0} \in(0,1]$ such that $\mathcal{N}^{t_{0}} \subset \tilde{\mu}_{p}\left(\tilde{N}_{p}\right)$ for every $p \in \Sigma_{i}$. Observe that $\left.\mathcal{E}\right|_{\mathcal{N}^{t_{0}}}$ is conjugate to $\left.\mathcal{E}\right|_{\mathcal{N}}$ by the suitable homothety $h$. Set $N_{p}=\tilde{\mu}_{p}^{-1}\left(\mathcal{N}^{t_{0}}\right)$ and $\mu_{p}=h \tilde{\mu}_{p}: N_{p} \rightarrow \mathcal{N}$. Then, $N_{p}$ is the requested neighborhood with its linearizing homeomorphism $\mu_{p}$. This finishes the proof of Theorem 2 .

\section{Proof of the classification theorem}

Let us prove that the diffeomorphisms $f$ and $f^{\prime}$ in $G(M)$ are topologically conjugate if and only if there is a homeomorphism $\hat{\varphi}: \hat{V}_{f} \rightarrow \hat{V}_{f^{\prime}}$ such that 
(1) $\eta_{f}=\eta_{f^{\prime}} \hat{\varphi}_{*}$

(2) $\hat{\varphi}\left(\hat{\Gamma}_{f}^{s}\right)=\hat{\Gamma}_{f^{\prime}}^{s}$ and $\hat{\varphi}\left(\hat{\Gamma}_{f}^{u}\right)=\hat{\Gamma}_{f^{\prime}}^{u}$.

\subsection{Necessity}

Let $f: M \rightarrow M$ and $f^{\prime}: M \rightarrow M$ be two elements in $G(M)$ which are topologically conjugated by some homeomorphism $h: M \rightarrow M$. Then $h$ conjugates the invariant manifolds of periodic points of $f$ and $f^{\prime}$. More precisely, if $p$ is a periodic point of $f$, then $h(p)$ is a periodic point of $f^{\prime}$ and $h\left(W^{u}(p)\right)=W^{u}(h(p)), h\left(W^{s}(p)\right)=W^{s}(h(p))$. In particular, $h$ maps $V_{f}$ to $V_{f^{\prime}}$ by a homeomorphism noted $\varphi$. Moreover, if $x$ is any points of $V_{f}$, for every $n \in \mathbb{Z}$ the following holds:

$$
\varphi\left(f^{n}(x)\right)=f^{\prime n}(\varphi(x)) .
$$

This formula says exactly that $\varphi$ is the lift of a map $\hat{\varphi}: \hat{V}_{f} \rightarrow \hat{V}_{f^{\prime}}$. By construction of $\eta_{f}$, the same formula says that $\eta_{f}=\eta_{f^{\prime}} \circ \hat{\varphi}_{*}$, where $\hat{\varphi}_{*}: H_{1}\left(\hat{V}_{f} ; \mathbb{Z}\right) \rightarrow H_{1}\left(\hat{V}_{f^{\prime}} ; \mathbb{Z}\right)$ denotes the map induced in homology. By definition of the quotient topology, $\hat{\varphi}$ is continuous. Since the same holds for $\varphi^{-1}$, one checks that $\hat{\varphi}$ is a homeomorphism. As $\varphi$ conjugates the laminations $\Gamma_{f}^{s}$ (resp. $\Gamma_{f}^{u}$ ) to $\Gamma_{f^{\prime}}^{s}\left(\operatorname{resp} . \Gamma_{f^{\prime}}^{u}\right.$ ), the same holds for $\hat{\varphi}$ in the quotient spaces with respect the projections of the laminations.

\subsection{Sufficiency}

For proving the sufficiency of the conditions in Theorem 1, let us consider a homeomorphism $\hat{\varphi}: \hat{V}_{f} \rightarrow \hat{V}_{f^{\prime}}$ such that:

(1) $\eta_{f}=\eta_{f^{\prime}} \hat{\varphi}_{*}$;

(2) $\hat{\varphi}\left(\hat{\Gamma}_{f}^{s}\right)=\hat{\Gamma}_{f^{\prime}}^{s}$ and $\hat{\varphi}\left(\hat{\Gamma}_{f}^{u}\right)=\hat{\Gamma}_{f^{\prime}}^{u}$.

From now on, the dynamical objects attached to $f^{\prime}$ will be denoted by $L^{\prime u}, L^{\prime s}, \Sigma_{i}^{\prime}$, . with the same meaning as $L^{u}, L^{s}, \Sigma_{i}, \ldots$ have with respect to $f$. By (1), $\hat{\varphi}$ lifts to an equivariant homeomorphism $\varphi: V_{f} \rightarrow V_{f^{\prime}}$, that is: $\left.f^{\prime}\right|_{V_{f^{\prime}}}=\left.\varphi f \varphi^{-1}\right|_{V_{f^{\prime}}}$ (for brevity, equivariance stands for $\left(f, f^{\prime}\right)$-equivariance). By (2), $\varphi$ maps $\Gamma_{f}^{u}$ to $\Gamma_{f^{\prime}}^{u}$ and $\Gamma_{f}^{s}$ to $\Gamma_{f^{\prime}}^{s}$. Thanks to Theorem 2 we may use compatible linearizable neighborhoods of the saddle points of $f$ (resp. $f^{\prime}$ ).

An idea of the proof is the following: we modify the homeomorphism $\varphi$ in a neighborhood of $\Gamma_{f}^{u}$ such that the final homeomorphism preserves the compatible foliations, then we do similar modification near $\Gamma_{f}^{s}$. So we get a homeomorphism $h: M \backslash\left(\Omega_{0} \cup \Omega_{3}\right) \rightarrow M \backslash\left(\Omega_{0}^{\prime} \cup \Omega_{3}^{\prime}\right)$ conjugating $\left.f\right|_{M \backslash\left(\Omega_{0} \cup \Omega_{3}\right)}$ with $\left.f^{\prime}\right|_{M \backslash\left(\Omega_{0}^{\prime} \cup \Omega_{3}^{\prime}\right)}$. Notice that $M \backslash\left(W_{\Omega_{1}}^{s} \cup W_{\Omega_{2}}^{s} \cup \Omega_{3}\right)=W_{\Omega_{0}}^{s}$ and $M \backslash\left(W_{\Omega_{1}^{\prime}}^{s} \cup W_{\Omega_{2}^{\prime}}^{s} \cup \Omega_{3}^{\prime}\right)=W_{\Omega_{0}^{\prime}}^{s}$. Since $h\left(W_{\Omega_{1}}^{s}\right)=W_{\Omega_{1}^{\prime}}^{s}$ and $h\left(W_{\Omega_{2}}^{s}\right)=W_{\Omega_{2}^{\prime}}^{s}$ then $h\left(W_{\Omega_{0}}^{s} \backslash \Omega_{0}\right)=$ $W_{\Omega_{0}^{\prime}}^{s} \backslash \Omega_{0}^{\prime}$. Thus for each connected component $A$ of $W_{\Omega_{0}}^{s} \backslash \Omega_{0}$ there is a sink $\omega \in \Omega_{0}$ such that $A=W_{\omega}^{s} \backslash \omega$. Similarly $h(A)$ is a connected component of $W_{\Omega_{0}^{\prime}}^{s} \backslash \Omega_{0}^{\prime}$ such that $h(A)=W_{\omega^{\prime}}^{s} \backslash \omega^{\prime}$ for a sink $\omega^{\prime} \in \Omega_{0}^{\prime}$. Then we can continuously extend $h$ to $\Omega_{0}$ assuming $h(\omega)=\omega^{\prime}$ for every $\omega \in \Omega_{0}$. A similar extension of $h$ to $\Omega_{3}$ finishes the proof. Thus below in a sequence of lemmas we explain only how to modify the homeomorphism $\varphi$ in a neighborhood of $\Gamma_{f}^{u}$ such that the final homeomorphism preserves the compatible foliations. 
Recall the partition $\Sigma_{0} \sqcup \cdots \sqcup \Sigma_{n}$ associated with the Smale order on the periodic points of index 2.

Lemma 4.1 For every $i=0, \ldots, n$ the following equality holds $\varphi\left(W_{i}^{u} \cap V_{f}\right)=W_{i}^{\prime u} \cap V_{f^{\prime}}$ and there is a unique continuous extension of $\left.\varphi\right|_{W_{i}^{u} \cap V_{f}}$ to $\Sigma_{i}$ which is equivariant and bijective from $\Sigma_{i}$ to $\Sigma_{i}^{\prime}$.

Proof: Let $p \in \Sigma_{0}$. Denote its orbit by $\operatorname{orb}_{f}(p)$. The punctured unstable manifold $W^{u}(p) \backslash\{p\}$ projects by $p_{f}$ to one compact leaf $\ell(p)$. Both sides of the next equality are $f$-invariant and project to the same leaf, thus:

$$
p_{f}^{-1}(\ell(p))=W^{u}(\operatorname{orb}(p)) \backslash\{\operatorname{orb}(p)\} .
$$

Then, the number of connected components of $p_{f}^{-1}(\ell(p))$ is $\operatorname{per}(p)$, the period of $p$. The image $\hat{\varphi}(\ell(p))$ is a compact leaf of $\hat{\Gamma}_{f^{\prime}}^{u}$. By the previous argument, it is $\ell^{\prime}\left(p^{\prime}\right)$ for some $p^{\prime} \in \Sigma_{0}^{\prime}$. Since $\hat{\varphi}$ lifts to $\varphi$, then $\varphi\left[p_{f}^{-1}(\ell(p))\right]=p_{f^{\prime}}^{-1}\left(\ell^{\prime}\left(p^{\prime}\right)\right)$ which implies the equality of the number of connected components. Thus $\operatorname{per}(p)=\operatorname{per}\left(p^{\prime}\right)$. From this, we can deduce that, up to replacing $p^{\prime}$ with $f^{\prime k}\left(p^{\prime}\right)$ for some integer $k$, we have $\varphi\left(W^{u}(p) \backslash\{p\}\right)=W^{u}\left(p^{\prime}\right) \backslash\left\{p^{\prime}\right\}$. Using the property $p=\lim _{n \rightarrow-\infty} f^{n}(x)$ for every $x \in W^{u}(p)$ and the similar property for $p^{\prime}$ in addition to the equivariance of $\varphi$, one extends continuously $\left.\varphi\right|_{W_{p}^{u}}$ by defining $\varphi(p)=p^{\prime}$. Doing the same for every orbit of $\Sigma_{0}$, we get a continuous extension of $\left.\varphi\right|_{W_{0}^{u}}$ to $\Sigma_{0}$ which is still equivariant. One easily checks that this extension is continuous, unique, and hence equivariant. Then, arguing similarly with $\hat{\varphi}^{-1}$, we derive that the extension of $\varphi$ maps $\Sigma_{0}$ bijectively onto $\Sigma_{0}^{\prime}$.

Denote $\ell_{0}:=\bigcup_{p \in \Sigma_{0}} \ell(p)$. We have $\hat{\varphi}\left(\ell_{0}\right)=\ell_{0}^{\prime}$. Let now $p \in \Sigma_{1}$. The closure in $\hat{V}_{f}$ of $\ell(p):=p_{f}\left(W^{u}(p) \backslash\{p\}\right)$ is contained in $\ell(p) \cup \ell_{0}$. We deduce that $\hat{\varphi}(\ell(p))$ is a leaf of $\hat{\Gamma}_{f^{\prime}}^{u}$ of the form $\ell^{\prime}\left(p^{\prime}\right)$ for some $p^{\prime} \in \Sigma_{1}^{\prime}$ and we can continue inductively. Thus, there is a continuous extension of $\left.\varphi\right|_{W_{i}^{u}}$ to every $\Sigma_{i}$ for $i=0,1, \ldots, n$ which is a bijection $\Sigma_{i} \rightarrow \Sigma_{i}^{\prime}$. Arguing with $\hat{\varphi}^{-1}$, we derive that $n^{\prime}=n$.

Recall the radial functions $r_{i}^{u}, r_{i}^{s}: N_{i} \rightarrow[0,+\infty)$ which are introduced above Definition 3.3 . recall also the order which is defined by $r_{i}^{s}$ on each stable separatrix $\gamma_{p}$ of $p \in \Sigma_{i}$. Analogous functions are associated with the dynamics of $f^{\prime}$.

Lemma 4.2 There is a unique continuous extension of $\left.\varphi\right|_{\Gamma_{f}^{u}}$

$$
\varphi^{u s}: \Gamma_{f}^{u} \cup\left(L^{u} \cap L^{s}\right) \longrightarrow \Gamma_{f^{\prime}}^{u} \cup\left(L^{\prime u} \cap L^{\prime s}\right)
$$

such that the following holds:

(1) If $x \in W_{j}^{u} \cap W_{i}^{s}, j>i$, then $\varphi^{u s}(x) \in W_{j}^{u} \cap W_{i}^{\prime s}$.

(2) If $x$ and $y$ lie in $\gamma_{p} \cap L^{u}$ with $r_{p}^{s}(x)<r_{p}^{s}(y)$, then $\varphi^{u s}(x)$ and $\varphi^{u s}(y)$ lie in $\gamma_{\varphi(p)}^{\prime} \cap L^{\prime u}$ with $r_{\varphi(p)}^{\prime s}\left(\varphi^{u s}(x)\right)<r_{\varphi(p)}^{s}\left(\varphi^{u s}(y)\right)$. 
Notice that $\left.\varphi\right|_{\Gamma_{f}^{u}}$ being equivariant, its continuous extension is also equivariant.

Proof: This statement is proved by induction on $i$. We recall that $V_{i} \backslash L_{i}^{s}=V_{f} \backslash \operatorname{cl}\left(W_{i}^{u}\right)$ is a dense open set in $V_{f}$ (and similarly with '), and according to Lemma 4.1, $\varphi$ maps $V_{i} \backslash L_{i}^{s}$ to $V_{i}^{\prime} \backslash L_{i}^{\prime s}$ homeomorphically and conjugates $f$ to $f^{\prime}$. Thus, for every $i=0, \ldots, n$, we have an equivariant homeomorphism $\varphi_{i}: V_{i} \backslash L_{i}^{s} \rightarrow V_{i}^{\prime} \backslash L_{i}^{\prime s}$ which maps $W_{j}^{u} \backslash L_{i}^{s}$ to $W_{j}^{\prime u} \backslash L_{i}^{\prime s}$ for every $j>i$, again as a consequence of Lemma 4.1 .

First, take $i=0$. The manifold $\hat{V}_{0}$ is closed and three-dimensional. We have $\hat{L}_{0}^{s}=\hat{W}_{0,0}^{s}$, which consists of finite number disjoint smooth circles, and similarly with'.

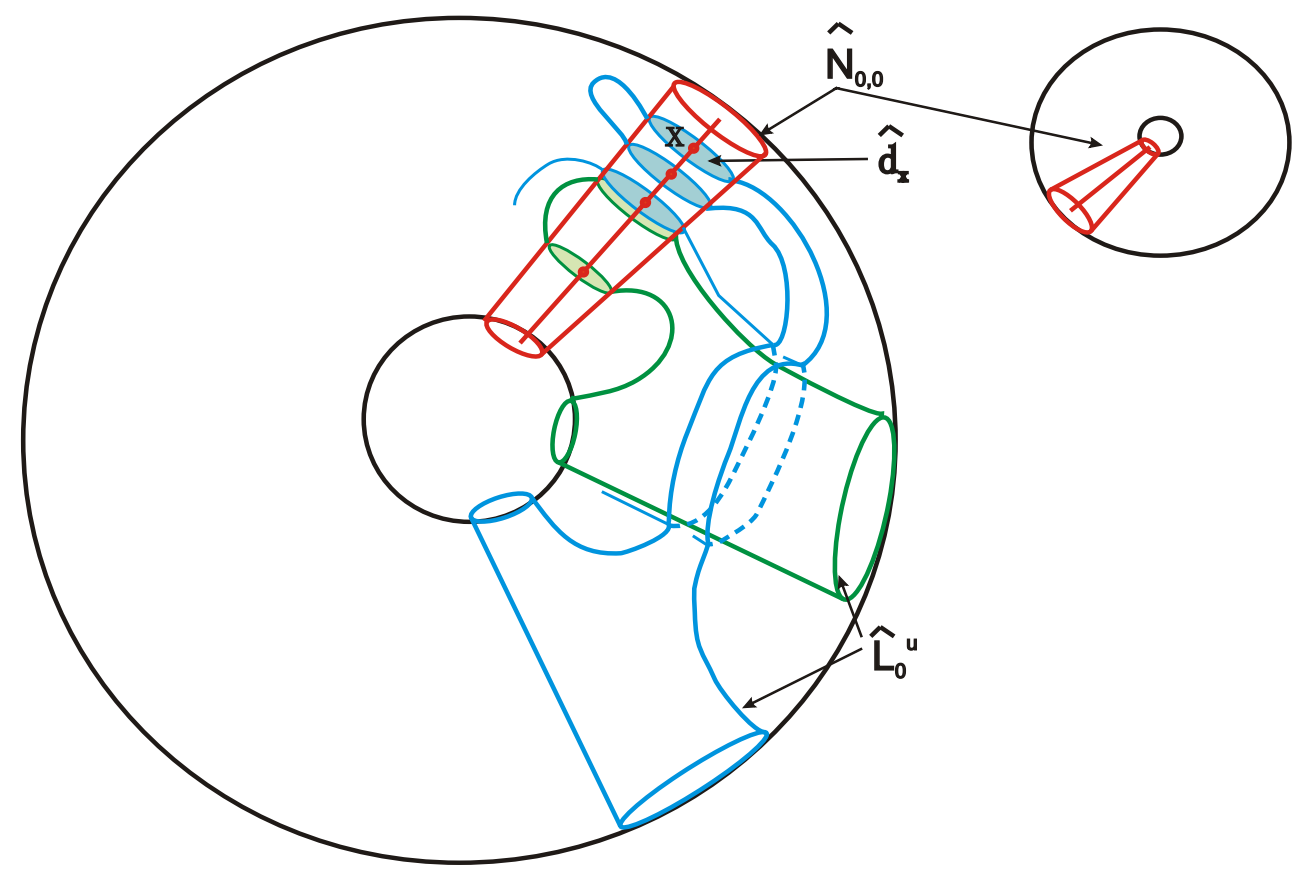

Figure 5: Case $i=0$ in proof of Lemma 4.2 for the diffeomorphism from Figure 3.

We look for an extension $\hat{\varphi}_{0}^{u s}$ of $\left.\hat{\varphi}_{0}\right|_{\hat{L}_{0}^{u}}$ to $\hat{L}_{0}^{s} \cap \hat{L}_{0}^{u}$. If $N_{0}$ is the neighborhood of $\Sigma_{0}$ extracted from a compatible system given by Theorem 2 and if $\hat{N}_{0,0}$ denotes the corresponding tubular neighborhood of $\hat{L}_{0}^{s}$ in $\hat{V}_{0}$, the trace of $\hat{L}_{0}^{u}$ in that tube is a lamination by disks:

$$
\hat{L}_{0}^{u} \cap \hat{N}_{0,0}=\left\{\hat{d}_{x} \mid x \in \hat{L}_{0}^{u} \cap \hat{L}_{0}^{s}\right\},
$$

where $\hat{d}_{x}$ denotes the fiber of the tube over $x \in \hat{L}_{0}^{s}$ (see figure 5 ).

The complement in $\hat{V}_{0}^{\prime}$ of the interior of $\hat{N}_{0,0}^{\prime}$ is a compact set contained in $\hat{V}_{0}^{\prime} \backslash \hat{L}_{0}^{\prime s}$. Then its preimage $K$ by the homeomorphism $\hat{\varphi}_{0}$ is a compact set contained in $\hat{V}_{0} \backslash \hat{L}_{0}^{s}$. When $t=0$, we have $\hat{N}_{0,0}^{t}=\hat{L}_{0}^{s}$ and hence disjoint from $K$. Then, if $t$ is small enough, $\hat{\varphi}_{0}\left(\hat{N}_{0,0}^{t} \backslash \hat{L}_{0}^{s}\right) \subset \hat{N}_{0,0}^{\prime} \backslash \hat{L}_{0}^{\prime s}$. Finally, the map $\hat{\varphi}_{0}$ (which is not defined on $\hat{L}_{0}^{s}$ ) possesses the two following properties:

1. If $N_{0}$ is shrunk enough, we have $\left(\hat{\varphi}_{0}\left(\hat{N}_{0,0}\right) \cap \hat{L}_{0}^{u}\right) \subset\left(\hat{N}_{0,0}^{\prime} \cap \hat{L}_{0}^{\prime u}\right)$, where $\hat{N}_{0,0}^{\prime}$ denotes the tube associated with the chosen linearizable neighborhood $N_{0}^{\prime}$ of $\Sigma_{0}^{\prime}$. 
2. If $\hat{d}_{x}$ is a plaque of $\hat{L}_{0}^{u} \cap \hat{N}_{0,0}$, the image $\hat{\varphi}_{0}\left(\hat{d}_{x} \backslash\{x\}\right)$ is contained in some fiber $\hat{d}_{x^{\prime}}$, with $x^{\prime} \in \hat{L}_{0}^{\prime u} \cap L_{0}^{\prime s}$.

As a consequence, the requested extension may be defined by $\hat{\varphi}_{0}^{u s}(x)=x^{\prime}$. As the considered plaques are arcwise connected, the construction lifts to the cover and yields a continuous map $\varphi_{0}^{u s}: \Gamma_{f}^{u} \cup\left(L_{0}^{s} \cap L_{0}^{u}\right) \rightarrow \Gamma_{f^{\prime}}^{u} \cup\left(L_{0}^{\prime s} \cap L_{0}^{\prime u}\right)$ which is a continuous equivariant extension of $\left.\varphi\right|_{\Gamma_{f}^{u}}$.

It remains to prove that $\varphi_{0}^{u s}$ is increasing on its domain in each separatrix of $\Sigma_{0}$. For this aim, consider a point $p \in \Sigma_{0}$, one of its separatrices $\gamma_{p}$ and a connected component $N_{\gamma_{p}}$ of $N_{p} \backslash W_{p}^{u}$ containing $\gamma_{p}$. Take an infinite proper arc $C$ in $N_{\gamma_{p}} \backslash W_{p}^{s}$ which crosses transversely each leaf of the foliation $F_{0}^{u}$ and which has one end in $p$. We orient $C$ so that its projection onto $\gamma_{p}$ is positive. Its image through $\varphi_{0}$ is a proper $\operatorname{arc} C^{\prime}$ contained in $N_{\varphi(p)}^{\prime} \backslash W_{\varphi(p)}^{\prime u}$. Moreover, $\varphi(p)$ is one end of $C^{\prime}$. For $x, y \in \gamma_{p} \cap L^{u}$, the inequality $r_{p}^{s}(x)<r_{p}^{s}(y)$ implies $r_{\varphi(p)}^{\prime s}\left(\varphi_{0}^{u s}(x)\right)<r_{\varphi(p)}^{\prime s}\left(\varphi_{0}^{u s}(y)\right)$ if we are sure that $C^{\prime}$ intersects each leaf of $L_{0}^{\prime u} \cap N_{\varphi(p)}^{\prime}$ at most in one point. That is true since $\varphi_{0}$ is a homeomorphism on its image from $N_{0} \backslash W_{0}^{s}$ to $N_{0}^{\prime} \backslash W_{0}^{\prime s}$ mapping $L_{0}^{u}$ into $L_{0}^{\prime u}$.

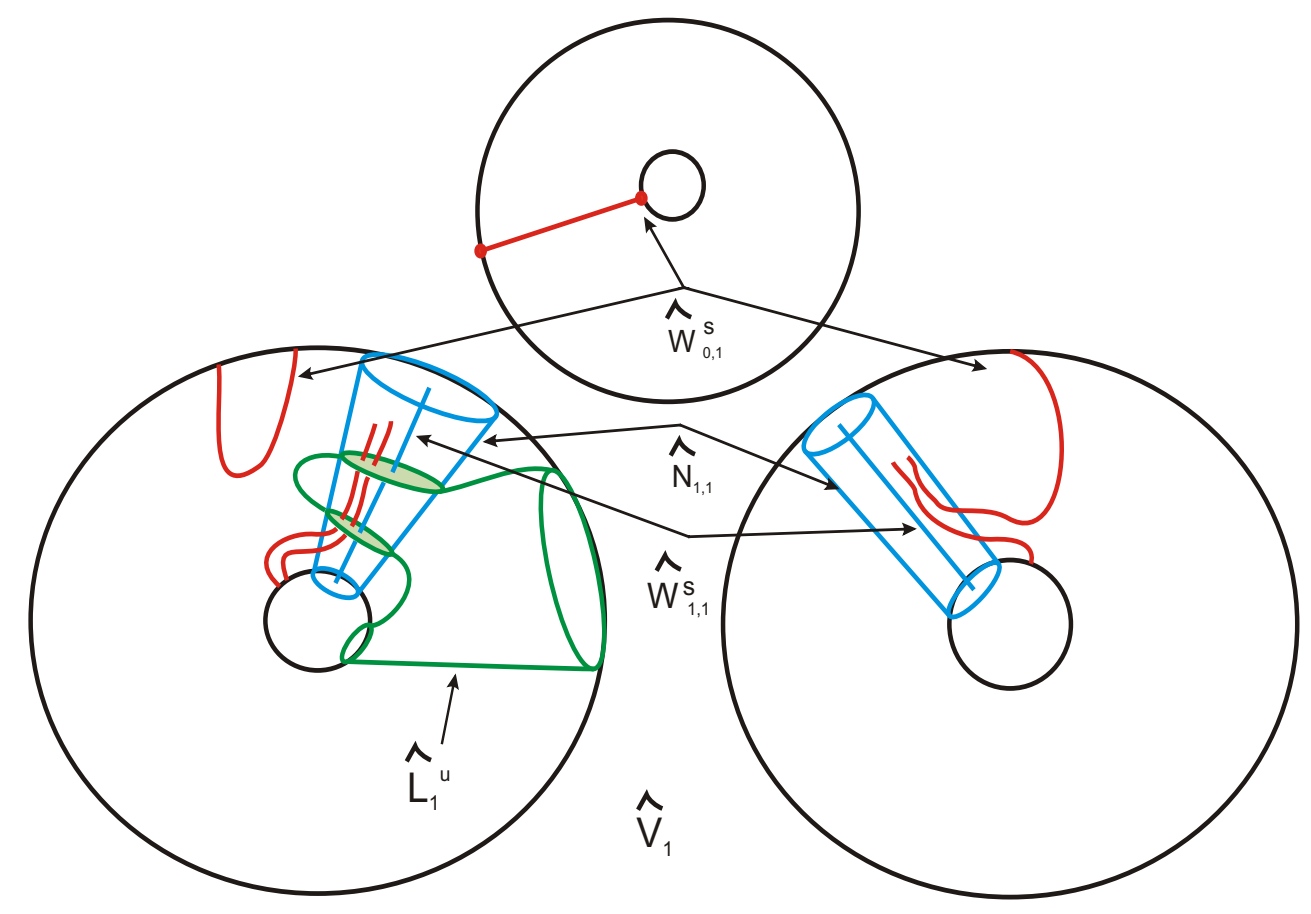

Figure 6: Illustration of induction in Lemma 4.2 for the diffeomorphism from Figure 3.

For the induction, let $i \in\{1, \ldots, n\}$ and let us assume that there is a continuous extension

$$
\varphi_{i-1}^{u s}: \Gamma_{f}^{u} \cup \bigcup_{j=0}^{i-1}\left(L_{j}^{s} \cap L_{j}^{u}\right) \rightarrow \Gamma_{f^{\prime}}^{u} \cup \bigcup_{j=0}^{i-1}\left(L_{j}^{\prime s} \cap L_{j}^{\prime u}\right),
$$

which is monotone on each separatrix of $\Sigma_{j}, j<i$. The image $\hat{W}_{i, i}^{s}$ of $W_{i}^{s}$ by the projection $p_{i}$ : $V_{i} \rightarrow \hat{V}_{i}$ is made of finitely many disjoint circles which are the images of the stable separatrices 
of $\Sigma_{i}$. About $\hat{W}_{i, i}^{s}$, there is a tube $\hat{N}_{i, i}$ which is the projection by $p_{i}$ of a neighborhood $N_{i}$ of $\Sigma_{i}$ extracted from a compatible system given by Theorem 2 (see Figure 6). The trace of $\hat{L}_{i}^{u}$ in that tube is a lamination by disks:

$$
\hat{L}_{i}^{u} \cap \hat{N}_{i, i}=\left\{\hat{d}_{x} \mid x \in \hat{L}_{i}^{u} \cap \hat{W}_{i, i}^{s}\right\},
$$

where $\hat{d}_{x}$ denotes the fiber of the tube over $x \in \hat{W}_{i, i}^{s}$.

In $V_{i}$, there are two laminations $L_{i}^{u}$ and $L_{i}^{s}$ (and the corresponding objects with '). The map $\varphi_{i}$, not defined on $L_{i}^{s}$, sends $L_{i}^{u} \backslash L_{i}^{s}$ homeomorphically onto $L_{i}^{\prime u} \backslash L_{i}^{\prime s}$. By the induction hypothesis, the restriction $\varphi_{i} \mid\left(L_{i}^{u} \backslash L_{i}^{s}\right)$ extends continuously to $L_{i}^{u} \backslash W_{i}^{s}$; this extension, automatically equivariant, is denoted by $\psi_{i}$. This induces on the quotient space $\hat{V}_{i}$ a homeomorphism

$$
\hat{\psi}_{i}: \hat{L}_{i}^{u} \backslash \hat{W}_{i, i}^{s} \rightarrow \hat{L}_{i}^{\prime u} \backslash \hat{W}_{i, i}^{\prime s} .
$$

In order to extend $\hat{\psi}_{i}$ to $\hat{L}_{i}^{u} \cap \hat{L}_{i}^{s}$, we use the fact that $\hat{L}_{i}^{u}$ is compact for arguing as in the case $i=0$. Consider the tube $\hat{N}_{i, i}^{t}$ depending on $t \in(0,1)$ and look at its compact lamination by disks $\hat{L}_{i}^{u} \cap \hat{N}_{i, i}^{t}$. After removing $\hat{W}_{i, i}^{s}$ which marks one puncture on each leaf, it is leaf-wise mapped by $\hat{\psi}_{i}$ into $\hat{V}_{i}^{\prime} \backslash \hat{W}_{i, i}^{\prime s}$. As in case $i=0$, the above-mentioned compactness allows us to conclude that there exists some $t \in(0,1)$ such that $\hat{L}_{i}^{u} \cap\left(\hat{N}_{i, i}^{t} \backslash \hat{W}_{i, i}^{s}\right)$ is mapped into $\hat{N}_{i, i}^{\prime}$ where $\hat{N}_{i, i}^{\prime}$ denotes the tube associated with the chosen linearizable neighborhood $N_{i}^{\prime}$ of $\Sigma_{i}^{\prime}$. Finally, the map $\hat{\psi}_{i}$ possesses the two following properties:

1. If $N_{i}$ is shrunk enough, we have $\left(\hat{\psi}_{i}\left(\hat{N}_{i, i}\right) \cap \hat{L}_{i}^{u}\right) \subset\left(\hat{N}_{i, i}^{\prime} \cap \hat{L}_{i}^{\prime u}\right)$.

2. If $\hat{d}_{x}$ is a plaque of $\hat{L}_{i}^{u} \cap \hat{N}_{i, i}$, the image $\hat{\psi}_{i}\left(\hat{d}_{x} \backslash\{x\}\right)$ is contained in some fiber $\hat{d}_{x^{\prime}}$, with $x^{\prime} \in \hat{L}_{i}^{\prime u} \cap W_{i}^{\prime s}$.

Now, the extension $\hat{\varphi}_{i}^{u s}$ of $\hat{\psi}_{i}$ is defined by $x \mapsto x^{\prime}$. One checks it is a continuous extension. The requested $\varphi_{i}^{u s}$ is the lift of $\hat{\varphi}_{i}^{u s}$ to $V_{i}$. It has the required properties allowing us to finish the induction.

Remark 4.3 Due to Lemma 3.2 we may assume that in all lemmas below the chosen values $t=\beta_{i}, a_{i}, \ldots$ are such that the boundary of the linearizable neighborhood $N_{i}^{t}$ does not contain any heteroclinic point.

Lemma 4.4 There are numbers $\beta_{0}, \ldots, \beta_{n} \in(0,1)$ such that, for every $i \in\{0, \ldots, n\}$, for every point $p \in \Sigma_{i}$ and $x \in N_{p}^{\beta_{i}} \cap L^{u}$, the following inequality holds:

$$
r_{i}^{\prime u}\left(\varphi^{u s}\left(x_{i}^{u}\right)\right) r_{i}^{\prime s}\left(\varphi^{u s}\left(x_{i}^{s}\right)\right)<\frac{1}{2} .
$$

Proof: As $N_{n} \cap L^{u}=W_{n}^{u}$ and $\varphi^{u s}\left(W_{n}^{u}\right)=W_{n}^{u}$, it is possible to chose any $\beta_{n} \in(0,1)$. 
Indeed, for $p \in \Sigma_{n}$ and $x \in W_{p}^{u}$, we have $r_{\varphi(p)}^{\prime s}\left(\varphi^{u s}\left(x_{i}^{s}\right)\right)=0$. For $i \in\{0, \ldots, n-1\}$ and $p \in \Sigma_{i}$, choose some heteroclinic point $y \in W_{p}^{s} \cap L^{u}$ arbitrarily. Set:

$$
\lambda_{p}^{\prime u}(t)=\sup _{x}\left\{r_{\varphi(p)}^{\prime u}\left(\varphi^{u s}\left(x_{i}^{u}\right)\right) \mid x \in N_{p}^{t} \cap F_{i, y}^{u}\right\} \quad \text { and } \quad \lambda_{p}^{\prime s}=r_{p}^{\prime s}\left(\varphi^{u s}(y)\right) .
$$

When $t$ goes to 0 , the arc $N_{p}^{t} \cap F_{i, y}^{u}$ shrinks to the point $y$. Then, according to Lemma 4.2 , $\lambda_{p}^{\prime u}(t)$ also goes to 0 . Therefore, there exists some $\beta_{p} \in(0,1)$ such that $\lambda_{p}^{\prime u}\left(\beta_{p}\right) \lambda_{p}^{\prime s}<\frac{1}{8}$. Denote by $Q_{p}$ the compact subset of $M$ bounded by $\partial N_{p}^{\beta_{p}}, F_{i, y}^{u}$ and $f^{p e r(p)}\left(F_{i, y}^{u}\right)$. Notice that $Q_{p}$ is a fundamental domain for the restriction of $f^{p e r(p)}$ to the connected component of $N_{p}^{\beta_{p}} \backslash W_{p}^{u}$ containing $y$. For every $x \in Q_{p}$, we have $r_{\varphi(p)}^{\prime u}\left(\varphi^{u s}\left(x_{i}^{u}\right)\right) \leq 4 \lambda_{p}^{\prime u}\left(\beta_{p}\right)$ and $r_{\varphi(p)}^{\prime s}\left(\varphi^{u s}\left(x_{i}^{s}\right)\right) \leq \lambda_{p}^{\prime s}$. Then, for every $x \in Q_{p} \cap L^{u}$ we have:

$$
r_{p}^{\prime u}\left(\varphi^{u s}\left(x_{i}^{u}\right)\right) r_{p}^{\prime s}\left(\varphi^{u s}\left(x_{i}^{s}\right)\right) \leq 4 \lambda_{p}^{\prime u}\left(\beta_{p}\right) \lambda_{p}^{\prime s}<\frac{1}{2} .
$$

Set $\beta_{i}=\min _{p \in \Sigma_{i}}\left\{\beta_{p}\right\}$. Hence, $\beta_{i}$ is the required number.

Lemma 4.5 When $n>0$, there exist real numbers $a_{j} \in\left(0, \beta_{j}\right]$ fulfilling the following property: for every $j=1, \ldots, n$ and every integer $i<j$, each connected component of $\hat{W}_{i, i}^{s} \cap \hat{N}_{j, i}^{a_{j}}$ is an open interval which is either disjoint from $A_{j}^{i}:=\bigcup_{k=i+1}^{j-1} \hat{N}_{k, i}^{a_{k}}$ or included in $A_{j}^{i}$. Moreover, only finitely many of these intervals are not covered by $A_{j}^{i}$.

Proof: The proof is done by induction on $j$ from 1 to $n$. For $j=1$, one is allowed to take $a_{1}=\beta_{1}$. Indeed, $\hat{W}_{0,0}^{s}$ is a smooth curve and $\hat{W}_{1,0}^{u}$ is a smooth closed surface which is transverse to $\hat{W}_{0,0}^{s}$. Therefore, there are finitely many intersection points. By the choice of $\beta_{1}$, the projection in $\hat{V}_{0}$ of $N_{1}^{a_{1}}$ is a tubular neighborhood of $\hat{W}_{1,0}^{u}$. Moreover, each component of $\hat{W}_{0,0}^{s} \cap \hat{N}_{1,0}^{a_{1}}$ is a fiber of this tube.

For the induction, assume the numbers $a_{1}, \ldots, a_{j-1}$ are given with the required properties and let us find $a_{j}$. In particular, the subset $A_{j}^{i}$ is assumed to be defined. According to Remark 4.3. the boundary of $A_{j}^{i}$ contains no heteroclinic point.

First, fix $i<j$. Consider the projection $\hat{W}_{j, i}^{u}$ of $W_{j}^{u}$ in $\hat{V}_{i}$. This is a union of leaves in the lamination $\hat{L}_{i}^{u}$. The following is a well-known fact (see, for example, Statement 1.1 in [12]): if $x$ is a point from $\hat{L}_{i}^{u}$ which is accumulated by a sequence of plaques from $\hat{W}_{j, i}^{u}$, then $x$ does not lie in $\hat{W}_{j, i}^{u}$ but belongs to some $\hat{W}_{k, i}^{u}$ with $k<j$. Then the part of $\hat{W}_{j, i}^{u}$ which is covered by $A_{j}^{i}$ contains every intersection points $\hat{W}_{i, i}^{s} \cap \hat{W}_{j, i}^{u}$ except finitely many of them. From this finiteness and the fact that $A_{j}^{i} \cap \hat{W}_{i, i}^{s} \cap \hat{W}_{j, i}^{u}$ is actually contained in $A_{j}^{i}$, an easy compactness argument allows us to find a positive number $a_{j}^{i}$ such that the collection of disjoint intervals made by $\hat{W}_{i, i}^{s} \cap \hat{N}_{j}^{a_{j}^{i}}$ fulfills the requested property with respect to the considered $i$. Indeed, let $B(t)$ be the closure of $\partial A_{j}^{i} \cap \hat{W}_{i, i}^{s} \cap \hat{N}_{j}^{t}$. The intersection $\bigcap_{k \in \mathbb{N}} B\left(\frac{1}{k}\right)$ is empty. Then $B(t)$ is empty when $t$ is small enough. 

ties.

By defining $a_{j}:=\inf \left\{a_{j}^{0}, \ldots, a_{j}^{j-1}\right\}$, we are sure that $\hat{N}_{j}^{a_{j}}$ satisfies all the requested proper-

The corollary below follows from Lemma 4.5 immediately.

Corollary 4.6 For each $i \in\{0, \ldots, n-1\}$ the intersection $\hat{W}_{i, i}^{s} \cap\left(\bigcup_{j=i+1}^{n} \hat{N}_{j, i}^{a_{j}}\right)$ consists of finitely many open arcs $\hat{I}_{1}^{i}, \ldots, \hat{I}_{r_{i}}^{i}$ such that, for each $l=1, \ldots, r_{i}$, the arc $\hat{I}_{l}^{i}$ is a connected component of $\hat{W}_{i, i}^{s} \cap \hat{N}_{j, i}^{a_{j}}$ for some $j>i$.

For brevity, for $i=0, \ldots, n$, we denote by $\varphi_{i}^{u}$ the restriction $\left.\varphi^{u s}\right|_{W_{i}^{u}}$ in the rest of the proof of Theorem 1. Let $\psi_{i}^{s}: W_{i}^{s} \rightarrow W_{i}^{s}$ be any equivariant homeomorphism which extends $\left.\varphi^{u s}\right|_{W_{i}^{s} \cap L^{u}}$ and let $t_{i} \in(0,1)$ be a small enough number so that, for every $x \in N_{i}^{t_{i}}$, the next inequality holds:

$$
(*)_{i} \quad r^{\prime s}\left(\varphi_{i}^{u}\left(x_{i}^{u}\right)\right) r^{\prime u}\left(\psi_{i}^{s}\left(x_{i}^{s}\right)\right)<1 .
$$

In this setting, one derives an equivariant embedding $\phi_{\varphi_{i}^{u}, \psi_{i}^{s}}: N_{i}^{t_{i}} \rightarrow N_{i}^{\prime}$ which is defined by sending $x \in N_{i}^{t_{i}}$ to $\left(\varphi_{i}^{u}\left(x_{i}^{u}\right), \psi_{i}^{s}\left(x_{i}^{s}\right)\right)$.

Lemma 4.7 There is an equivariant homeomorphism $\psi^{s}: L^{s} \rightarrow L^{\prime s}$ consisting of conjugating homeomorphisms $\psi_{0}^{s}: W_{0}^{s} \rightarrow W_{0}^{\prime s}, \ldots, \psi_{n}^{s}: W_{n}^{s} \rightarrow W_{n}^{\prime s}$ such that for each $i \in\{0, \ldots, n\}$ :

(1) $\left.\psi_{i}^{s}\right|_{W_{i}^{s} \cap L^{u}}=\left.\varphi_{i}^{u}\right|_{W_{i}^{s} \cap L^{u}}$;

(2) the topological embedding $\phi_{\varphi_{i}^{u}, \psi_{i}^{s}}$ is well-defined on $N_{i}^{a_{i}}$;

(3) if $x \in\left(W_{i}^{s} \cap N_{j}^{a_{j}}\right), j>i$, then $\psi_{i}^{s}(x)=\phi_{\varphi_{j}^{u}, \psi_{j}^{s}}(x)$.

Proof: We are going to construct $\psi_{i}^{s}$ by a decreasing induction on $i$ from $i=n$ to $i=0$. The stable manifolds of the saddles in $\Sigma_{n}$ have no heteroclinic points. Therefore, the only constraints on $\psi_{n}^{s}$ imposed by the first item is its value on $\Sigma_{n}$. In particular, we are allowed to change $\psi_{n}^{s}$ to $f^{\prime k} \circ \psi_{n}^{s}$ if $k$ is admissible in the sense that $k$ is a multiple of all periods $\operatorname{per}(p), p \in \Sigma_{n}$.

This remark is used in the following way. One starts with any equivariant homeomorphism $\psi_{n}^{s}$ such that for any $p \in \Sigma_{n}$ the stable manifold $W_{p}^{s}$ is mapped to the stable manifold of $\varphi_{n}^{u}(p)$; hence, item 1 is fulfilled. Choose a fundamental domain $I$ of $\left.f\right|_{W_{n}^{s} \backslash \Sigma_{n}}$. Consider the fundamental domain of $\left.f\right|_{N_{n}^{a_{n}} \backslash W_{n}^{u}}$ defined by $N_{I}:=\left\{x \in N_{n}^{a_{n}} \mid x_{n}^{s} \in I\right\}$; set $\lambda_{n}^{\prime u}:=\sup \left\{r^{\prime u}\left(\varphi_{n}^{u}\left(x_{n}^{u}\right) \mid x \in N_{I}\right\}\right.$ and $\lambda_{n}^{\prime s}:=\sup \left\{r^{\prime s}\left(\psi_{n}^{s}\left(x_{n}^{s}\right) \mid x \in N_{I}\right\}\right.$. If the product $\lambda_{n}^{\prime u} \lambda_{n}^{\prime s}$ is less than 1 , the inequality $(*)_{n}$ is fulfilled by the pair $\left(\varphi_{n}^{u}, \psi_{n}^{s}\right)$ and hence, the embedding $\phi_{\varphi_{n}^{u} \psi_{n}^{s}}$ is well-defined on $N_{n}^{a_{n}}$.

If not, we replace $\psi_{n}^{s}$ with $f^{\prime k} \circ \psi_{n}^{s}$ with $k$ admissible and large enough. Indeed, the effect of this change is to multiply $\lambda_{n}^{\prime s}$ by some positive factor bounded by $\left(\frac{1}{4}\right)^{k}$ while $\lambda_{n}^{\prime u}$ is kept fixed and hence, $(*)_{n}$ becomes fulfilled when $k$ is large enough. Since the third item is empty for $i=n$, we have built some $\psi_{n}^{s}$ as desired.

For the induction, let us build $\psi_{i}^{s}, i<n$, with the required properties assuming that the homeomorphisms $\psi_{n}^{s}, \ldots, \psi_{i+1}^{s}$ have already been built. The stable manifolds of saddles in $\Sigma_{i}$ have heteroclinic intersections with unstable manifolds of saddles in $\Sigma_{j}$ with $j>i$ only. The 
image $\hat{W}_{i, i}^{s}$ of $W_{i}^{s}$ under $p_{i}: V_{i} \rightarrow \hat{V}_{i}$ is a closed smooth 1-dimensional submanifold. According to Corollary 4.6. the intersection $\hat{W}_{i, i}^{s} \cap\left(\bigcup_{j=i+1}^{n} \hat{N}_{j}^{a_{j}}\right)$ consists of finitely many open $\operatorname{arcs} \hat{I}_{1}^{i}, \ldots, \hat{I}_{r_{i}}^{i}$ such that $\hat{I}_{l}^{i}$ for each $l=1, \ldots, r_{i}$ is a connected component of $\hat{W}_{i, i}^{s} \cap \hat{N}_{j, i}^{a_{j}}$ for some $j>i$.

In order to satisfy the third item of the statement, $\psi_{i}^{s}$ is defined on $p_{i}^{-1}\left(\hat{I}_{l}^{i}\right)$ in an equivariant way. Denote by $\psi_{i, l}^{s}$ this partial definition of $\psi_{i}^{s}$; its image is contained in $W_{i, i}^{\prime s}$.

More precisely, if $I_{l, \alpha}^{i}$ is a connected component of $p_{i}^{-1}\left(\hat{I}_{l}^{i}\right)$ it is a proper arc in some $N_{i}^{a_{j}}$ and it intersects $W_{j}^{u}$ in a unique point $x_{l, \alpha}^{i}$. Set $x_{l, \alpha}^{i}=\varphi^{u s}\left(x_{l, \alpha}^{i}\right)$ and denote $I_{l, \alpha}^{i}$ the connected component of $W_{i}^{\prime s} \cap N_{j}^{\prime}$ passing through the point $x_{l, \alpha}^{i}$. Then, the restriction of $\psi_{i, l}^{s}$ to the arc $I_{l, \alpha}^{i}$ reads:

$$
\psi_{i, l, \alpha}^{s}=\left.\phi_{\varphi_{j}^{u}, \psi_{j}^{s}}\right|_{I_{l, \alpha}^{i}}: I_{l, \alpha}^{i} \rightarrow I_{l, \alpha}^{i} .
$$

By Lemma 4.2, the map $\varphi^{u s}$ sends $W_{i}^{s} \cap L^{u}$ to $W_{i}^{\prime s} \cap L^{\prime u}$ preserving the order on each separatrix of $W_{i}^{s} \backslash \Sigma_{i}$ and $W_{i}^{\prime s} \backslash \Sigma_{i}^{\prime}$. On the other hand, $\psi_{i, l, \alpha}^{s}$ is also order preserving. Both together imply that $\psi_{i, l}^{s}$ is order preserving since we know that it is an injective map. Moreover, the union of all $\psi_{i, l}^{s}$ - which makes sense as their respective domains are mutually disjoint - is order preserving. Therefore, there is an equivariant homeomorphism $\psi_{i}^{s}: W_{i}^{s} \backslash \Sigma_{i} \rightarrow W_{i}^{s} \backslash \Sigma_{i}$ which extends all $\psi_{i, l}^{s}$.

Since $\varphi^{u s}$ is continuous, the above homeomorphism extends continuously to $\psi_{i}^{s}: W_{i}^{s} \rightarrow W_{i}^{s}$. At this point of the construction items 1 and 3 of the statement are satisfied. The condition of item 2 follows from Lemma 4.4 for stable separatrices that contain heteroclinic points. If some stable separatrix has no heteroclinic points, one changes $\psi_{i}^{s}$ to $f^{\prime k} \circ \psi_{i}^{s}$ on the separatrix where $k$ is a large common multiple of the period of the separatrix, like to the construction made in the case $i=n$.

Proof of Theorem 1 Continued. Let us recall that we denoted by $\mathcal{E}: \mathbb{R}^{3} \rightarrow \mathbb{R}^{3}$ the canonical linear diffeomorphism with the unique fixed point $O=(0,0,0)$ which is a saddle point whose unstable manifold is the plane $O x_{1} x_{2}$ and stable manifold is the axis $O x_{3}$; for simplicity, we assume that $\mathcal{E}$ has a sign $\nu=+$ (see the beginning of Section 3). Let

$$
N=\left\{\left(x_{1}, x_{2}, x_{3}\right) \in \mathbb{R}^{3}: 0 \leq\left(x_{1}^{2}+x_{2}^{2}\right) x_{3} \leq 1\right\} .
$$

Let $\rho>0, \delta \in\left(0, \frac{\rho}{4}\right)$ and

$$
\begin{gathered}
d=\left\{\left(x_{1}, x_{2}, x_{3}\right) \in \mathbb{R}^{3}: x_{1}^{2}+x_{2}^{2} \leq \rho^{2}, x_{3}=0\right\}, \\
U=\left\{\left(x_{1}, x_{2}, x_{3}\right) \in \mathbb{R}^{3}:(\rho-\delta)^{2} \leq x_{1}^{2}+x_{2}^{2} \leq \rho^{2}, x_{3}=0\right\}, \\
c=\left\{\left(x_{1}, x_{2}, x_{3}\right) \in \mathbb{R}^{3}: x_{1}^{2}+x_{2}^{2}=\rho^{2}, x_{3}=0\right\}, \\
c^{0}=\left\{\left(x_{1}, x_{2}, x_{3}\right) \in \mathbb{R}^{3}: x_{1}^{2}+x_{2}^{2}=\left(\rho-\frac{\delta}{2}\right)^{2}, x_{3}=0\right\}, \\
c^{1}=\left\{\left(x_{1}, x_{2}, x_{3}\right) \in \mathbb{R}^{3}: x_{1}^{2}+x_{2}^{2}=(\rho-\delta)^{2}, x_{3}=0\right\} .
\end{gathered}
$$

Let $K=d \backslash$ int $\mathcal{E}^{-1}(d), V=(K \cup \mathcal{E}(K)) \cap\left\{\left(x_{1}, x_{2}, x_{3}\right) \in \mathbb{R}^{3}: x_{1} \geq 0, x_{3}=0\right\}$ and $\beta=U \cap O x_{1}^{+}$, where $O x_{1}^{+}=\left\{\left(x_{1}, x_{2}, x_{3}\right) \in \mathbb{R}^{3}: x_{3}^{2}+x_{2}^{2}=0, x_{1}>0\right\}$. 
Choose a point $Z^{0}=\left(0,0, z^{0}\right) \in O x_{3}^{+}$such that $\rho^{2} z^{0}<\frac{1}{4}$ (see Figure 7 ). Then, choose a point $Z^{1}=\left(0,0, z^{1}\right)$ in $O x_{3}^{+}$so that $z^{0}>z^{1}>\frac{z^{0}}{4}$. Let $\Pi(z)=\left\{\left(x_{1}, x_{2}, x_{3}\right) \in \mathbb{R}^{3}: x_{3}=z\right\}$. In what follows, for every subset $A \subset O x_{1} x_{2}$, we denote by $\tilde{A}$ will denote the cylinder $\tilde{A}=A \times\left[0, z^{0}\right]$. Denote by $\mathcal{W}$ the 3 -ball bounded by the annulus $\tilde{c}$ and the two planes $\Pi\left(z^{0}\right)$ and $\Pi\left(\frac{z^{0}}{4}\right)$. Let $\Delta$ be a closed 3-ball bounded by the surface $\tilde{c}^{1}$ and the two planes $O x_{1} x_{2}$ and $\Pi\left(z^{1}\right)$. Let

$$
\mathcal{T}=\bigcup_{k \in \mathbb{Z}} \mathcal{E}^{k}(\tilde{d}) \quad \text { and } \quad \mathcal{H}=\bigcup_{k \in \mathbb{Z}} \mathcal{E}^{k}(\Delta)
$$

Notice that the construction yields $\mathcal{H} \subset$ int $\mathcal{T}$ and makes $\mathcal{W}$ a fundamental domain for the action of $\mathcal{E}$ on $\mathcal{T}$.

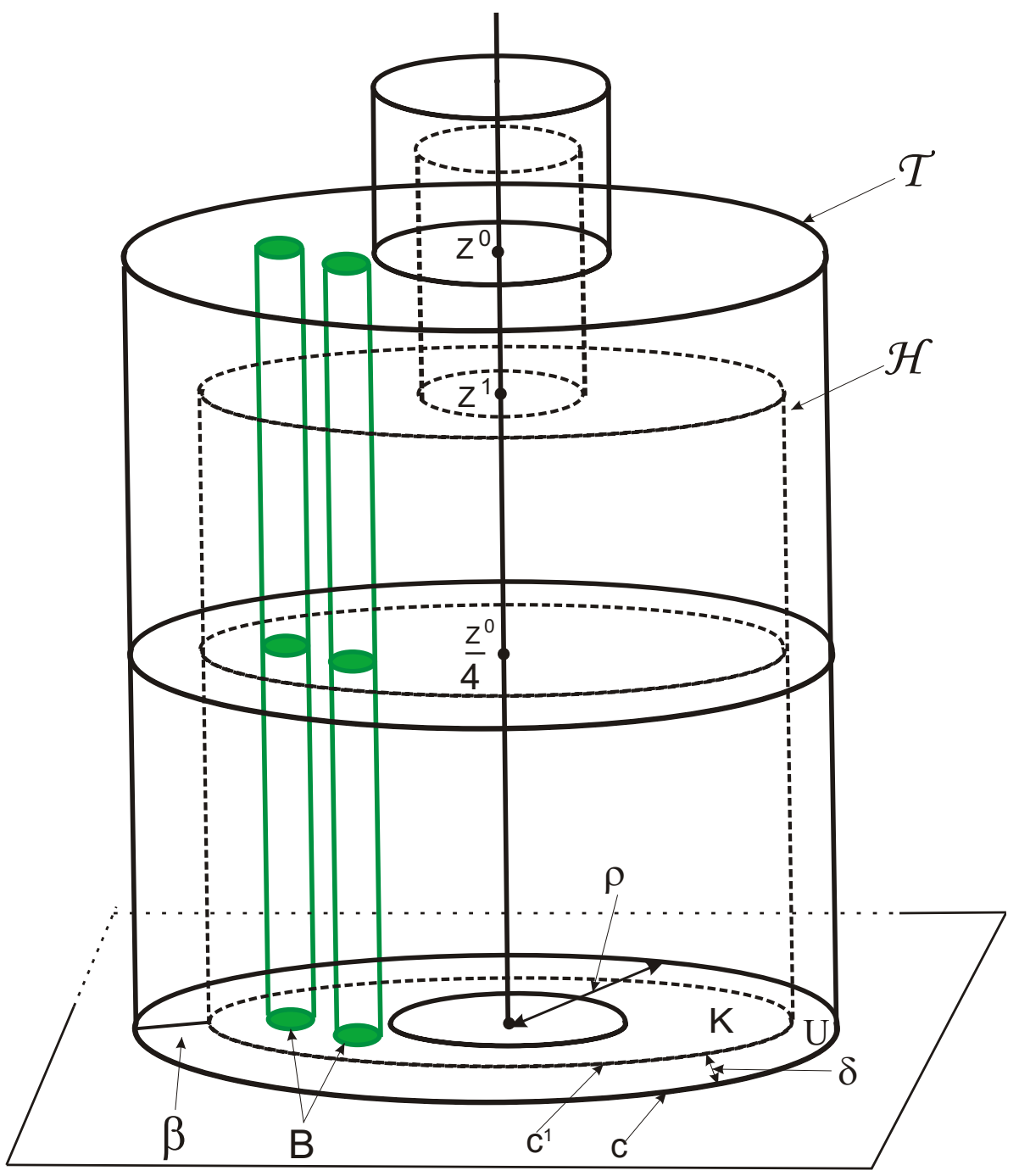

Figure 7: A linear model

Now, we come back to $f$ and construct some neighborhoods $\mathcal{H}_{\gamma} \subset \mathcal{T}_{\gamma}$ around each separatrix $\gamma$ which contains heteroclinic points. Therefore, we consider only the case $n \geq 1$ and separatrices 
of the saddle points from $\Sigma_{i}$ for $i \in\{0, \ldots, n-1\}$, but not those from $\Sigma_{n}$ since their onedimensional separatrices do not contain heteroclinic points. Let $G_{i}$ be the union of all stable separatrices of saddle points in $\Sigma_{i}$ that contain heteroclinic points. Let $\check{G}_{i} \subset G_{i}$ be the union of separatrices in $G_{i}$ such that $G_{i}=\bigcup_{\gamma \in \breve{G}_{i}} \operatorname{orb}(\gamma)$ and, for every pair $\left(\gamma_{1}, \gamma_{2}\right)$ of distinct separatrices in $\check{G}_{i}$ and every $k \in \mathbb{Z}$, one has $\gamma_{2} \neq f^{k}\left(\gamma_{1}\right)$. For $\gamma \in G_{i}$ with the end point $p \in \Sigma_{i}$ and a point $q \in \Sigma_{j}, j>i$, let us consider a sequence of different periodic orbits $p=p_{0} \prec p_{1} \prec \ldots \prec p_{k}=q$ such that $\gamma \cap W_{p_{1}}^{u} \neq \emptyset$, the length of the longest such chain is denoted by $\operatorname{beh}(q \mid \gamma)$.

Let $\gamma \in \check{G}_{i}$ be a separatrix of $p \in \Sigma_{i}$ and let $N_{\gamma}^{t}$ be the connected component of $N_{p}^{t} \backslash W_{p}^{u}$ which contains $\gamma$. We endow with the index $\gamma$ (resp. $p$ ) the preimages in $M$ (through the linearizing map $\mu_{p}$ ) of all objects from the linear model $\mathcal{N}$ associated with the separatrix $\gamma$ (resp. $p$ ); for being precise we decide that $\mu_{p}(\gamma)=O x_{3}^{+}$. For a separatrix $\gamma$ in $\check{G}_{i}$, let us fix a saddle point $q_{\gamma}$ such that $b e h\left(q_{\gamma} \mid \gamma\right)=1$. Notice that the intersection $\gamma \cap W_{q_{\gamma}}^{u}$ consists of a finite number of heteroclinic orbits.

Lemma 4.8 Let $n \geq 1, i \in\{0, \ldots, n-1\}$. For every $\gamma \in \check{G}_{i}$ there are positive numbers $\rho, \delta$ and $\varepsilon$ (depending on $\gamma$ ) such that for every heteroclinic point $Z_{\gamma}^{0} \in\left(\gamma \cap W_{q_{\gamma}}^{u}\right)$ with $z^{0}<\varepsilon$ the following properties hold:

(1) $U_{p}$ avoids all heteroclinic points;

(2) $\varphi\left(\tilde{d}_{p}\right) \subset \phi_{\varphi_{i}^{u}, \psi_{i}^{s}}\left(N_{i}^{a_{i}}\right)$;

(3) $\varphi\left(\tilde{c}_{p}\right) \cap \phi_{\varphi_{i}^{u}, \psi_{i}^{s}}\left(\tilde{c}_{p}^{0}\right)=\emptyset, \varphi\left(\tilde{c}_{p}^{1}\right) \cap \phi_{\varphi_{i}^{u}, \psi_{i}^{s}}\left(\tilde{c}_{p}^{0}\right)=\emptyset$ and $\varphi\left(\tilde{\beta}_{\gamma}\right) \subset \phi_{\varphi_{i}^{u}, \psi_{i}^{s}}\left(\tilde{V}_{\gamma}\right)$.

Proof: Let $\gamma \in \check{G}_{i}, i \in\{0, \ldots, n-1\}$. Due to Lemma 3.2 , there is a generic $\rho>0$ such that the curve $c_{\gamma}$ avoids all heteroclinic points. Since $W_{l}^{s}$ accumulates on $W_{k}^{s}$ for every $l<k$, then $K_{p} \cap W_{i-1}^{s}$ is made of a finite number of heteroclinic points $y_{1}, \ldots, y_{r}$ which we can cover by closed 2-discs $b_{1}, \ldots, b_{r} \subset$ int $K_{p}$. In $K_{p} \backslash \operatorname{int}\left(b_{1} \cup \ldots \cup b_{r}\right)$ there is a finite number of heteroclinic points from $W_{i-2}^{s}$ which we cover by the union of a finite number of closed 2-discs, and so on. Thus we get that all heteroclinic points in $K_{p}$ belong to the union of finitely many closed 2-discs avoiding $\partial K_{p}$. Therefore, there is $\delta \in\left(0, \frac{\rho}{4}\right)$ such that $U_{p}$ avoids heteroclinic points. This proves item (1).

By assumption of Theorem 1, $\varphi$ is defined on the complement of the stable manifolds and, by definition, $\phi_{\varphi_{i}^{u}, \psi_{i}^{s}}$ coincides with $\varphi$ on $W_{i}^{u} \backslash L^{s}$, and hence on $U_{p}$. As $\varphi$ and $\phi_{\varphi_{i}^{u}, \psi_{i}^{s}}$ are continuous, we can choose $\varepsilon>0$ sufficiently small so that, if $Z_{\gamma}^{0}$ is any heteroclinic point in the intersection $\gamma \cap W_{q_{\gamma}}^{u}$ with $z^{0}<\varepsilon$, the requirements of (2) and (3) are fulfilled.

Let us fix $U_{p}$ satisfying item (1) of Lemma 4.8 and define

$$
U_{i}=\bigcup_{p \in \Sigma_{i}}\left(\bigcup_{k=0}^{p e r(p)-1} f^{k}\left(U_{p}\right)\right), \quad K_{i}=\bigcup_{p \in \Sigma_{i}}\left(\bigcup_{k=0}^{p e r(p)-1} f^{k}\left(K_{p}\right)\right) .
$$

Until the end of Section 4, we assume that for every $\gamma \in \check{G}_{n-1}$ the neighborhoods $\mathcal{T}_{\gamma}$ and $\mathcal{H}_{\gamma}$ have the parameters $\rho, \delta, \varepsilon, z^{0}$ as in Lemmas 4.8 and $z^{1}$ is chosen such that the $\operatorname{arc}\left(z_{\gamma}^{0}, z_{\gamma}^{1}\right) \subset \gamma$ does not contain heteroclinic points. But, when $\gamma \in \check{G}_{i}, i \in\{0, \ldots, n-2\}$, the parameter $\varepsilon$ will be still more specified in Lemma 4.9 below. 
Lemma 4.9 Let $n \geq 2$. For every $i \in\{0, \ldots, n-2\}$ and $\gamma \in \check{G}_{i}$, there is a heteroclinic point $Z_{\gamma}^{0} \in \gamma$ satisfying the conditions of Lemma 4.8 and in addition:

$$
\mathcal{T}_{\gamma} \cap \tilde{U}_{j}=\emptyset \quad \text { for } \quad j \in\{i+1, \ldots, n-1\} .
$$

In this statement, it is meant that $\tilde{U}_{n-1}$ is associated with the points $Z_{\gamma^{\prime}}^{0}, \gamma^{\prime} \in \check{G}_{n-1}$ given by Lemma 4.8 and $\tilde{U}_{j}$ is associated with the points $Z_{\gamma^{\prime \prime}}^{0}, \gamma^{\prime \prime} \in \check{G}_{j}$ given by Lemma 4.9 for every $j>i$. Therefore, it makes sense to prove Lemma 4.9 by decreasing induction on $i$ from $i=n-2$ to 0 . This is what is done below.

Proof: Let us first prove the lemma for $i=n-2$. Let $\gamma \in \check{G}_{n-2}$ and let $p$ be the saddle end point of $\gamma$. Notice that the intersection $\gamma \cap K_{n-1}$ consists of a finite number points $a_{1}, \ldots, a_{l}$ avoiding $U_{n-1}$. Let $d_{1}, \ldots, d_{l} \subset K_{n-1}$ be compact discs with centres $a_{1}, \ldots, a_{l}$ and radius $r_{*}$ (in linear coordinates of $N_{p}$ ) avoiding $U_{n-1}$. Let us choose a number $n^{*} \in \mathbb{N}$ such that $\frac{\rho}{2^{n^{*}}}<r^{*}$. Let $Z_{\gamma}^{*} \subset \gamma$ be a point such that the segment $\left[p, Z_{\gamma}^{*}\right]$ of $\gamma$ avoids $\tilde{K}_{n-1}$ and $\mu_{p}\left(Z_{\gamma}^{*}\right)=Z^{*}=\left(0,0, z^{*}\right)$ where $z^{*}<\varepsilon$. Then every heteroclinic point $z_{\gamma}^{0}$ so that $z^{0}<\frac{z^{*}}{2^{n^{*}}}$ possesses the property: $\mathcal{T}_{\gamma} \cap \tilde{K}_{n-1}$ avoids $\tilde{U}_{n-1}$.

For the induction, let us assume now that the construction of the desired heteroclinic points is done for $i+1, i+2, \ldots, n-2$. Let us do it for $i$. Let $\gamma \in \check{G}_{i}$. By assumption of the induction $\left(\bigcup_{k=i+1}^{j-1} \mathcal{T}_{k}\right) \cap \tilde{U}_{j}=\emptyset$ for $j \in\{i+2, \ldots, n-1\}$. Since $W_{k-1}^{s}$ accumulates on $W_{k}^{s}$ for every $k \in\{0, \ldots, n\}$, then $\left(\bigcup_{k=i+1}^{j-1} \mathcal{T}_{k}\right) \cap K_{j}$ is a compact subset of $K_{j}$ and the intersection $\left(\gamma \backslash\left(\bigcup_{k=i+1}^{j-1} \mathcal{T}_{k}\right)\right) \cap K_{j}$ consists of a finite number points $a_{1}, \ldots, a_{l}$ avoiding $U_{j}$. Let $d_{1}, \ldots, d_{l} \subset K_{j}$ be compact discs with centres $a_{1}, \ldots, a_{l}$ and radius $r_{*}$ (in linear coordinates of $N_{p}$ ) avoiding $U_{j}$ and such that $r_{*}$ is less than the distance between $\partial\left(K_{j} \backslash U_{j}\right)$ and $\left(\bigcup_{k=i+1}^{j-1} \mathcal{T}_{k}\right) \cap K_{j}$. Similar to the case $i=n-2$ it is possible to choose a heteroclinic point $Z_{\gamma}^{0}$ sufficiently close to the saddle $p$ where $\gamma$ ends such that the set $\left(\mathcal{T}_{\gamma} \backslash\left(\bigcup_{k=i+1}^{j-1} \mathcal{T}_{k}\right)\right) \cap \tilde{K}_{j}$ avoids $\tilde{U}_{j}$.

In what follows, we assume that, for every $\gamma \subset \breve{G}_{i}, i \in\{0, \ldots, n-2\}$, the neighborhoods $\mathcal{T}_{\gamma}$ and $\mathcal{H}_{\gamma}$ have parameters $\rho, \delta, \varepsilon, z^{0}$ as in Lemma 4.8 and moreove $\varepsilon$ satisfies to Lemma 4.9 . For $i \in\{0, \ldots, n-1\}$, we set

$$
\mathcal{T}_{i}=\bigcup_{\gamma \subset \breve{G}_{i}}\left(\bigcup_{k=0}^{p e r(\gamma)-1} f^{k}\left(\mathcal{T}_{\gamma}\right)\right)
$$

For $\gamma \subset \check{G}_{i}, j>i$, let us denote by $\mathcal{J}_{\gamma, j}$ the union of all connected components of $W_{j}^{u} \cap \mathcal{T}_{\gamma}$ 
which do not lie in int $\mathcal{T}_{k}$ with $i<k<j$. Let $\mathcal{J}_{\gamma}=\bigcup_{j=i+1}^{n} \mathcal{J}_{\gamma, j}$ and

$$
\mathcal{J}_{i}=\bigcup_{\gamma \subset \breve{G}_{i}}\left(\bigcup_{k=0}^{p e r(\gamma)-1} f^{k}\left(\mathcal{J}_{\gamma}\right)\right)
$$

Let $\mathcal{W}_{\gamma}$ be the fundamental domain of $\left.f^{p e r(\gamma)}\right|_{\mathcal{T}_{\gamma} \backslash W_{p}^{u}}$ limited by the plaques of the two heteroclinic points $Z_{\gamma}^{0}$ and $f^{p e r(\gamma)} Z_{\gamma}^{0}$. Notice that $\gamma \cap \mathcal{W}_{\gamma}$ is a fundamental domain of $\left.f^{p e r(\gamma)}\right|_{\gamma}$. Since $W_{k}^{u}$ accumulates on $W_{l}^{u}$ only when $l<k$, then the set $\mathcal{J}_{\gamma, j} \cap \mathcal{W}_{\gamma}$ consists of a finite number of closed 2-discs. Hence, the set $\mathcal{J}_{\gamma} \cap \gamma \cap \mathcal{W}_{\gamma}$ consists of a finite number of heteroclinic points; denote them $Z_{\gamma}^{2}, \ldots, Z_{\gamma}^{m}(m$ depends on $\gamma)$. Finally, choose an arbitrary point $Z_{\gamma}^{1} \in \gamma$ so that the $\operatorname{arc}\left(z_{\gamma}^{0}, z_{\gamma}^{1}\right) \subset \gamma$ does not contain heteroclinic points from $\mathcal{J}_{\gamma}$. Let us construct $\mathcal{H}_{\gamma}$ using the point $Z^{1}=\mu_{p}\left(Z_{\gamma}^{1}\right)$ and the parameter $\delta$ from Lemma 4.8 . Without loss of generality we will assume that $\mu_{p}\left(Z_{\gamma}^{i}\right)=Z^{i}=\left(0,0, z^{i}\right)$ for $z^{0}>z^{1}>\ldots>z^{m}>\frac{z^{0}}{4}$. For $i=0, \ldots, n-1$ let

$$
\mathcal{H}_{i}=\bigcup_{\gamma \subset \breve{G}_{i}}\left(\bigcup_{k=0}^{p e r(\gamma)-1} f^{k}\left(\mathcal{H}_{\gamma}\right)\right) \quad \text { and } \quad \mathcal{M}_{i}=V_{f} \cup \bigcup_{k=0}^{i}\left(G_{k} \cup \Sigma_{k}\right) \text {. }
$$

Lemma 4.10 There is an equivariant topological embedding $\varphi_{0}: \mathcal{M}_{0} \rightarrow M^{\prime}$ with following properties:

(1) $\varphi_{0}$ coincides with $\varphi$ out of $\mathcal{T}_{0}$;

(2) $\left.\varphi_{0}\right|_{\mathcal{H}_{0}}=\left.\phi_{\psi_{0}^{u}, \psi_{0}^{s}}\right|_{\mathcal{H}_{0}}$, where $\psi_{0}^{u}=\left.\varphi\right|_{W_{0}^{u}}$;

(3) $\varphi_{0}\left(W_{1}^{u}\right)=W_{1}^{\prime u}$ and $\varphi_{0}\left(W_{k}^{u} \backslash \bigcup_{j=1}^{k-1}\right.$ int $\left.\mathcal{T}_{j}\right) \subset W_{k}^{\text {uu }}$ for every $k \in\{2, \ldots, n\}$.

Proof: The desired $\varphi_{0}$ should be an interpolation between $\varphi: V_{f} \backslash \mathcal{T}_{0} \rightarrow M^{\prime}$ and $\left.\phi_{\varphi_{0}^{u}, \psi_{0}^{s}}\right|_{\mathcal{H}_{0}}$. Due to Lemma 4.8 (2) and the equivariance of the considered maps, the embedding

$$
\xi_{0}=\phi_{\psi_{0}^{u}, \psi_{0}^{s}}^{-1} \varphi: \mathcal{T}_{0} \backslash W_{0}^{s} \rightarrow M
$$

is well-defined. Let $\gamma \subset \check{G}_{0}$ be a separatrix ending at $p \in \Sigma_{0}$ and $\xi_{\gamma}=\left.\xi_{0}\right|_{\mathcal{T}_{\gamma}}$. By construction, the topological embedding $\xi=\mu_{p} \xi_{\gamma} \mu_{p}^{-1}: \mathcal{T} \rightarrow N$ has the following properties:

(i) $\xi \mathcal{E}=\mathcal{E} \xi\left(\right.$ as $\mathcal{E} \mu_{p}=\mu_{p} f^{\operatorname{per}(\gamma)}$ and $\left.\xi_{\gamma} f^{p e r(\gamma)}=f^{\operatorname{per}(\gamma)} \xi_{\gamma}\right)$;

(ii) $\xi$ is the identity on $O x_{1} x_{2}\left(\right.$ as $\left.\left.\phi_{\psi_{0}^{u}, \psi_{0}^{s}}\right|_{W_{0}^{u}}=\left.\varphi\right|_{W_{0}^{u}}\right)$;

(iii) $\xi\left(\Pi\left(z^{0}\right) \cap \mathcal{T}\right) \subset \Pi\left(z^{0}\right)$ and $\xi\left(\Pi\left(z^{i}\right) \cap \partial \mathcal{T}\right) \subset \Pi\left(z^{i}\right), i \in\{2, \ldots, m\}\left(\right.$ as $\left.\xi_{\gamma}\left(L^{u} \cap \mathcal{T}_{\gamma} \backslash \gamma\right) \subset L^{u}\right)$;

(iv) $\xi(\tilde{c}) \cap \tilde{c}^{0}=\emptyset, \xi\left(\tilde{c}^{1}\right) \cap \tilde{c}^{0}=\emptyset$ and $\xi(\tilde{\beta}) \subset \tilde{V}$ (due to Lemma 4.8 (3)).

Thus, $\xi$ satisfies all conditions of Proposition 5.1 and, hence, there is an embedding $\zeta: \mathcal{T} \rightarrow N$ such that:

(I) $\zeta a=a \zeta$;

(II) $\zeta$ is the identity on $\mathcal{H}$;

(III) $\zeta\left(\Pi\left(z^{i}\right) \cap \mathcal{T}\right) \subset \Pi\left(z^{i}\right), i \in\{0,2, \ldots, m\}$ 
(IV) $\zeta$ is $\xi$ on $\partial \mathcal{T}$.

Then the embedding $\zeta_{\gamma}=\mu_{p}^{-1} \zeta \mu_{p}: \mathcal{T}_{\gamma} \rightarrow N_{\gamma}$ satisfies the properties:

(I') $\zeta_{\gamma} f^{\text {per }(\gamma)}=f^{\text {per }(\gamma)} \zeta_{\gamma}$;

(II') $\zeta_{\gamma}$ is the identity on $\mathcal{H}_{\gamma}$;

(III') $\zeta\left(\mathcal{J}_{\gamma}\right) \subset L^{u}$

(IV') $\zeta_{\gamma}$ is $\xi_{\gamma}$ on $\partial \mathcal{T}_{\gamma}$.

Independently, one does the same for every separatrix $\gamma \subset \check{G}_{0}$. Then, it is extend to all separatrices in $G_{0}$ by equivariance. As a result, we get a homeomorphism $\zeta_{0}$ of $\mathcal{T}_{0}$ onto $\xi_{0}\left(\mathcal{T}_{0}\right)$ which coincides with $\xi_{0}$ on $\partial \mathcal{T}_{0}$. Now, define the embedding $\varphi_{0}: \mathcal{M}_{0} \rightarrow M^{\prime}$ to be equal to

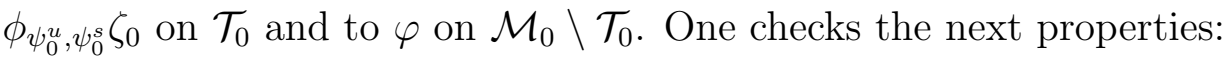

(1) $\varphi_{0}$ coincides with $\varphi$ out of $\mathcal{T}_{0}$;

(2) $\left.\varphi_{0}\right|_{\mathcal{H}_{0}}=\left.\phi_{\psi_{0}^{u}, \psi_{0}^{s}}\right|_{\mathcal{H}_{0}}$

$\left(3^{\prime}\right) \varphi_{0}\left(\mathcal{J}_{0}\right) \subset L^{u}$.

The last property and the definition of the set $\mathcal{J}_{\gamma}$ imply that $\varphi_{0}\left(W_{1}^{u}\right)=W_{1}^{u}$ and $\varphi_{0}\left(W_{k}^{u} \backslash\right.$ $\left.\bigcup_{j=1}^{k-1} i n t \mathcal{T}_{j}\right) \subset W_{k}^{\prime u}$ for every $k \in\{2, \ldots, n\}$. Thus $\varphi_{0}$ satisfies all required conditions of the lemma.

Lemma 4.11 Assume $n \geq 2, i \in\{0, \ldots, n-2\}$, and assume there is an equivariant topological embedding $\varphi_{i}: \mathcal{M}_{i} \rightarrow M^{\prime}$ with following properties:

(1) $\varphi_{i}$ coincides with $\varphi_{i-1}$ out of $\mathcal{T}_{i}$;

(2) $\left.\varphi_{i}\right|_{\mathcal{H}_{i}}=\phi_{\psi_{i}^{u}, \psi_{i}^{s}}$, where $\psi_{i}^{u}=\left.\varphi_{i-1}\right|_{W_{i}^{u}}$ and $\varphi_{-1}=\varphi$;

(3) there is an $f$-invariant union of tubes $\mathcal{B}_{i} \subset\left(\mathcal{T}_{i} \cap \bigcup_{j=0}^{i-1} \mathcal{H}_{j}\right)$ containing $\left(\mathcal{T}_{i} \cap\left(\bigcup_{j=0}^{i-1} W_{j}^{s}\right)\right)$ where $\varphi_{i}$ coincides with $\varphi_{i-1}$ (we assume $\mathcal{B}_{0}=\emptyset$ );

(4) $\varphi_{i}\left(W_{i+1}^{u}\right)=W_{i+1}^{\prime u}$ and $\varphi_{i}\left(W_{k}^{u} \backslash \bigcup_{j=i+1}^{k-1} \operatorname{int} \mathcal{T}_{j}\right) \subset W_{k}^{\prime u}$ for every $k \in\{i+2, \ldots, n\}$.

Then there is a homeomorphism $\varphi_{i+1}$ with the same properties (1)-(4)

Proof: The desired $\varphi_{i+1}$ should be an interpolation between $\varphi_{i}: \mathcal{M}_{i+1} \backslash \mathcal{T}_{i+1} \rightarrow M^{\prime}$ and $\phi_{\psi_{i+1}^{u}}^{u},\left.\psi_{i+1}^{s}\right|_{\mathcal{H}_{i+1}}$ where $\psi_{i+1}^{u}=\left.\varphi_{i}\right|_{W_{i+1}^{u}}$. Let $\gamma \subset \check{G}_{i+1}$ be a separatrix ending at $p \in \Sigma_{i+1}$. It follows from the definition of the set $\mathcal{J}_{i}$ and the choice of the point $q_{\gamma}$ that $\left(W_{q_{\gamma}}^{u} \cap \mathcal{T}_{i}\right) \subset \mathcal{J}_{i}$. Then, due to condition (4) for $\varphi_{i}$ we have $\varphi_{i}\left(W_{q_{\gamma}}^{u} \cap \mathcal{T}_{i}\right) \subset W_{q^{\prime}}^{u}$. By the property (1) of the homeomorphism $\varphi_{i}$ and the properties of $\mathcal{T}_{i+1}$ from Lemmas 4.8 (1) and 4.9, we get that $\left.\varphi_{i}\right|_{\tilde{U}_{p}}=\left.\varphi\right|_{\tilde{U}_{p}}$. Then $\left.\phi_{\varphi_{i+1}^{u}, \psi_{i+1}^{s}}\right|_{\tilde{U}_{p}}=\left.\phi_{\psi_{i+1}^{u}, \psi_{i+1}^{s}}\right|_{\tilde{U}_{p}}$. Thus it follows from the property (2) in Lemma 4.8 that the following embedding is well-defined: $\xi_{\gamma}=\phi_{\psi_{i+1}^{u}, \psi_{i+1}^{s}}^{-1} \varphi_{i}: \mathcal{T}_{\gamma} \backslash(\gamma \cup p) \rightarrow M^{\prime}$.

By construction, the topological embedding $\xi=\mu_{p} \xi_{\gamma} \mu_{p}^{-1}$ satisfies to all conditions of Proposition 5.1. Let $\zeta$ be the embedding which is yielded by that proposition. Define $\zeta_{\gamma}=\mu_{p}^{-1} \zeta \mu_{p}$. Notice that by the property (3) of the homeomorphism $\psi^{s}$ in Lemma 4.7 and by the properties $\psi_{i+1}^{u}=\left.\varphi_{i}\right|_{W_{i}^{u}}$, we have that $\zeta_{\gamma}$ is the identity on a neighborhood $\tilde{B}_{\gamma} \subset\left(\mathcal{T}_{\gamma} \cap \bigcup_{j=0}^{i} \mathcal{H}_{j}\right)$ of 
$\mathcal{T}_{\gamma} \cap\left(\bigcup_{j=0}^{i} W_{j}^{s}\right)$. Independently, one does the same for every separatrix $\gamma \subset \check{G}_{i+1}$. Assuming that $\zeta_{f(\gamma)}=f^{\prime} \zeta_{\gamma} f^{-1}$ and $\tilde{B}_{i+1}=\bigcup_{\gamma \subset \tilde{G}_{i+1}}\left(\bigcup_{k=0}^{p e r(\gamma)-1} f^{k}\left(\tilde{B}_{\gamma}\right)\right)$ we get a homeomorphism $\zeta_{i+1}$ on $\mathcal{T}_{i+1}$. Thus the required homeomorphism coincides with $\phi_{\psi_{i+1}^{u}, \psi_{i+1}^{s}}$ on $\mathcal{H}_{i+1}$ and with $\varphi_{i}$ out of $\mathcal{T}_{i+1}$. $\diamond$

Let $G$ be the union of all stable one-dimensional separatrices which do not contain heteroclinic points, $N_{G}^{t}=\bigcup_{\gamma \subset G} N_{\gamma}^{t}$ and

$$
\mathcal{M}=\mathcal{M}_{n-1} \cup G \text {. }
$$

Lemma 4.12 There are numbers $0<\tau_{1}<\tau_{2}<1$ and an equivariant embedding $h_{\mathcal{M}}: \mathcal{M} \rightarrow$ $M^{\prime}$ with the following properties:

(1) $h_{\mathcal{M}}$ coincides with $\varphi_{n-1}$ out of $N_{G}^{\tau_{2}}$;

(2) $h_{\mathcal{M}}$ coincides with $\phi_{\varphi_{n-1}, \psi^{s}}$ on $\left.\right|_{\mathcal{N}_{G}^{\tau_{1}}}$, where $\psi^{s}: L^{s} \rightarrow L^{\prime s}$ is yielded by Lemma 4.7;

(3) there is an $f$-invariant neighborhood of the set $N_{G} \cap\left(G_{0} \cup \ldots \cup G_{n-1}\right)$ where $h_{\mathcal{M}}$ coincides with $\varphi_{n-1}$.

Proof: Let $\check{G} \subset G$ be a union of separatrices from $G$ such that $\gamma_{2} \neq f^{k}\left(\gamma_{1}\right)$ for every $\gamma_{1}, \gamma_{2} \subset \check{G}, k \in \mathbb{Z} \backslash\{0\}$ and $G=\bigcup_{\gamma \in \breve{G}} \operatorname{orb}(\gamma)$. Let $i \in\{0, \ldots, n\}, p \in \Sigma_{i}$ and $\gamma \subset G$.

Notice that $\left(N_{\gamma} \backslash(\gamma \cup p)\right) / f^{\operatorname{per}(\gamma)}$ is homeomorphic to $X \times[0,1]$ where $X$ is 2-torus and the natural projection $\pi_{\gamma}: N_{\gamma} \backslash(\gamma \cup p) \rightarrow X \times[0,1]$ sends $\partial N_{\gamma}^{t}$ to $X \times\{t\}$ for each $t \in(0,1)$ and sends $W_{p}^{u} \backslash p$ to $X \times\{0\}$. Let $\xi_{\gamma}=\left.\phi_{\left.\varphi_{n-1}\right|_{W_{i}^{u}}, \psi_{i}^{s}}^{-1} \varphi_{n-1}\right|_{N_{\gamma}^{a_{i}} \backslash(\gamma \cup p)}$ and $\hat{\xi}_{\gamma}=\left.\pi_{\gamma} \xi_{\gamma} \pi_{\gamma}^{-1}\right|_{X \times\left[0, a_{i}\right]}$. Due to item (3) of Lemma 4.11, the homeomorphism $\hat{\xi}_{\gamma}$ coincides with the identity in some neighborhood of $\pi_{\gamma}\left(N_{\gamma}^{a_{i}} \cap\left(G_{0} \cup \ldots \cup G_{n-1}\right)\right)$. Let us choose this neighborhood of the form $B_{\gamma} \times\left[0, a_{i}\right]$. Let us choose numbers $0<\tau_{1, \gamma}<\tau_{2, \gamma}<a_{i}$ such that $\hat{\xi}_{\gamma}\left(X \times\left[0, \tau_{2, \gamma}\right]\right) \subset X \times\left[0, \tau_{1, \gamma}\right)$. By construction, $\hat{\xi}_{\gamma}: X \times\left[0, \tau_{2, \gamma}\right] \rightarrow X \times[0,1]$ is a topological embedding which is the identity on $X \times\{0\}$ and $\left.\hat{\xi}_{\gamma}\right|_{B_{\gamma} \times\left[0, \tau_{2, \gamma}\right]}=\left.i d\right|_{B_{\gamma} \times\left[0, \tau_{2, \gamma}\right]}$. Then, by Proposition 5.2.

1. there is a homeomorphism $\hat{\zeta}_{\gamma}: X \times\left[0, \tau_{2, \gamma}\right] \rightarrow \hat{\xi}\left(X \times\left[0, \tau_{2, \gamma}\right]\right)$ such that $\hat{\zeta}_{\gamma}$ is identity on $X \times\left[0, \tau_{1, \gamma}\right]$ and is $\hat{\xi}_{\gamma}$ on $X \times\left\{\tau_{2, \gamma}\right\}$.

2. $\left.\hat{\zeta}_{\gamma}\right|_{B_{\gamma} \times\left[0, \tau_{2, \gamma}\right]}=\left.i d\right|_{B_{\gamma} \times\left[0, \tau_{2, \gamma}\right]}$.

Let $\zeta_{\gamma}$ be a lift of $\hat{\zeta}_{\gamma}$ on $N_{\gamma}^{\tau_{2, \gamma}}$ which $\xi_{\gamma}$ on $\partial N_{\gamma}^{\tau_{2, \gamma}}$. Thus $\varphi_{\gamma}=\phi_{\left.\varphi_{n-1}\right|_{W_{i}^{u}}, \psi_{i}^{s}} \zeta_{\gamma}$ is the desired extension of $\varphi_{n-1}$ to $N_{\gamma}$. Doing the same for every separatrix $\gamma \subset \check{G}$ and extending it to the other separatrices from $G$ by equivariance, we get the required homeomorphism $h_{\mathcal{M}}$ for $\tau_{1}=\min _{\gamma \subset \breve{G}}\left\{\tau_{1, \gamma}\right\}$ and $\tau_{2}=\min _{\gamma \subset \breve{G}}\left\{\tau_{2, \gamma}\right\}$.

So far in this section, we have modified the homeomorphism $\varphi$ in a union of suitable linearizable neighborhoods of $\Omega_{2}$ (with their 1-dimensional separatrices removed) so that the modified homeomorphism extends equivariantly to $W^{s}\left(\Omega_{2}\right)$. At the same time, we can perform a similar modification about $\Omega_{1}$ since the involved linearizable neighborhoods of $\Omega_{2}$ and $\Omega_{1}$ are 
mutually disjoint. Thus, we get a homeomorphism $h: M \backslash\left(\Omega_{0} \cup \Omega_{3}\right) \rightarrow M \backslash\left(\Omega_{0}^{\prime} \cup \Omega_{3}^{\prime}\right)$ conjugating $\left.f\right|_{M \backslash\left(\Omega_{0} \cup \Omega_{3}\right)}$ with $\left.f^{\prime}\right|_{M \backslash\left(\Omega_{0}^{\prime} \cup \Omega_{3}^{\prime}\right)}$. Notice that $M \backslash\left(W_{\Omega_{1}}^{s} \cup W_{\Omega_{2}}^{s} \cup \Omega_{3}\right)=W_{\Omega_{0}}^{s}$ and $M \backslash\left(W_{\Omega_{1}^{\prime}}^{s} \cup W_{\Omega_{2}^{\prime}}^{s} \cup \Omega_{3}^{\prime}\right)=W_{\Omega_{0}^{\prime}}^{s}$. Since $h\left(W_{\Omega_{1}}^{s}\right)=W_{\Omega_{1}^{\prime}}^{s}$ and $h\left(W_{\Omega_{2}}^{s}\right)=W_{\Omega_{2}^{\prime}}^{s}$, then $h\left(W_{\Omega_{0}}^{s} \backslash \Omega_{0}\right)=W_{\Omega_{0}^{\prime}}^{s} \backslash \Omega_{0}^{\prime}$. Thus for each connected component $A$ of $W_{\Omega_{0}}^{s} \backslash \Omega_{0}$, there is a sink $\omega \in \Omega_{0}$ such that $A=W_{\omega}^{s} \backslash \omega$. Similarly, $h(A)$ is a connected component of $W_{\Omega_{0}^{\prime}}^{s} \backslash \Omega_{0}^{\prime}$ such that $h(A)=W_{\omega^{\prime}}^{s} \backslash \omega^{\prime}$ for a sink $\omega^{\prime} \in \Omega_{0}^{\prime}$. Then we can continuously extend $h$ to $\Omega_{0}$ by defining $h(\omega)=\omega^{\prime}$ for every $\omega \in \Omega_{0}$. A similar extension of $h$ to $\Omega_{3}$ finishes the proof of Theorem 1 .

\section{Topological background}

We use below the notations which have been introduced before Lemma 4.8 .

Proposition 5.1 Let $z^{0}>z^{1}>\ldots>z^{m}>\frac{z^{0}}{4}>0$ and $\xi: \mathcal{T} \backslash O x_{3} \rightarrow N$ be a topological embedding with the following properties:

(i) $\xi \mathcal{E}=\mathcal{E} \xi$

(ii) $\xi$ is the identity on $O x_{1} x_{2}$;

(iii) $\xi\left(\Pi\left(z^{0} \cap \mathcal{T}\right)\right)=\Pi\left(z^{0}\right)$ and $\xi\left(\Pi\left(z^{i}\right) \cap \partial \mathcal{T}\right) \subset \Pi\left(z^{i}\right), i \in\{2, \ldots, m\}$;

(iv) $\xi(\tilde{c}) \cap \tilde{c}^{0}=\emptyset, \xi\left(\tilde{c}^{1}\right) \cap \tilde{c}^{0}=\emptyset$ and $\xi(\tilde{\beta}) \subset \tilde{V}$.

Then there is a homeomorphism $\zeta: \mathcal{T} \rightarrow N$ such that

(I) $\zeta \mathcal{E}=\mathcal{E} \zeta$;

(II) $\zeta$ is the identity on $\mathcal{H}$ - and consequentively on $O x_{1} x_{2}$;

(III) $\zeta\left(\Pi\left(z^{i}\right) \cap \mathcal{T}\right) \subset \Pi\left(z^{i}\right), i \in\{0,2, \ldots, m\}$

(IV) $\zeta$ is $\xi$ on $\partial \mathcal{T}$.

Moreover, if $\xi$ is identity on $\tilde{B}$ for a set $B \subset(K \backslash U)$ then $\zeta$ is also identity on $\tilde{B}$.

Proof: For $j=0, \ldots, m$, we denote by $E_{j}$ the domain of $\mathbb{R}^{3}$ located between the horizontal planes $\Pi\left(z_{j}\right)$ and $\Pi\left(z_{j+1}\right)$, with $z_{m+1}=\frac{z_{0}}{4}$. Since the requested $\zeta$ has to be equivariant with respect to $\mathcal{E}$, it is useful to describe a fundamental domain $\mathcal{V}$ for the action of $\mathcal{E}$ on the closure of $\mathcal{T} \backslash \mathcal{H}$; the natural one is

$$
\mathcal{V}=\operatorname{cl}(\mathcal{T} \backslash \mathcal{H}) \cap\left(\bigcup_{j=0}^{m} E_{j}\right),
$$

where $\mathrm{cl}(-)$ stands for closure of $(-)$. The domain $\mathcal{V}$ is sliced by the horizontal planes $\Pi\left(z_{j}\right), j=$ $2, \ldots, m$, and the vertical cylinders $\mathcal{E}^{-1}(\tilde{c})$ and $\tilde{c}^{1}$, yielding the decomposition $\mathcal{V}=R_{0} \cup R_{1} \cup$ $Q_{0} \cup Q_{2} \cup \ldots \cup Q_{m}$ into solid tori whose interiors are mutually disjoint. Notice that the plane $\Pi\left(z_{1}\right)$ is not used in this decomposition.

More precisely, $R_{0} \subset E_{0}$ is limited by the cylinders $\mathcal{E}^{-1}\left(\tilde{c}^{1}\right)$ and $\mathcal{E}^{-1}(\tilde{c})$; then, $R_{1} \subset E_{0}$ is limited by the cylinders $\mathcal{E}^{-1}(\tilde{c})$ and $\tilde{c}^{1}$. The others of the list are obtained from $\tilde{U}$ by slicing $\mathcal{V}$ with horizontal planes. The first of the latter, namely $Q_{0}$, is special as it is bounded by $\Pi\left(z_{0}\right)$ and $\Pi\left(z_{2}\right)$; then, $Q_{j}$ is bounded by $\Pi\left(z_{j}\right)$ and $\Pi\left(z_{j+1}\right)$ for $j=2, \ldots, m$. The vertical parts in the boundaries of the above-mentioned solid tori are provided by the vertical slices or the vertical parts of $\partial \mathcal{T} \cup \partial \mathcal{H}$. 
For $j=0,2, \ldots, m$, let $U\left(z_{j}\right):=\tilde{U} \cap \Pi\left(z_{j}\right)$. By construction, the top face of $R_{0}$ is $U^{\prime}\left(z_{0}\right):=\mathcal{E}^{-1}\left(U\left(z_{m+1}\right)\right)=\Pi\left(z_{0}\right) \cap \mathcal{E}^{-1}(\tilde{U})$; its bottom is $U^{\prime}\left(z_{1}\right):=\Pi\left(z_{1}\right) \cap \mathcal{E}^{-1}(\tilde{U})$. Similarly, the top of $R_{1}$ is $U^{\prime \prime}\left(z_{0}\right):=\Pi\left(z_{0}\right) \cap \tilde{K}$ and its bottom is $U^{\prime \prime}\left(z_{1}\right):=\Pi\left(z_{1}\right) \cap \tilde{K}$.

It is important that each horizontal or vertical annulus Ann from the previous list is marked with a special arc noted $\beta(A n n)$ linking the two boundary components of Ann and defined as follows:

$$
\beta(A n n)=\text { Ann } \cap\left\{x_{1}>0, x_{2}=0\right\} .
$$

According to assumption (iv), all these arcs (except when $A n n=U^{\prime \prime}\left(z_{0}\right)$ or $U^{\prime \prime}\left(z_{1}\right)$ ) fulfill the next condition, referred to as the $\beta$-condition, namely: they are mapped by $\xi$ into $\left\{x_{1}>0\right\}$.

First of all, we define $\left.\zeta\right|_{R_{1}}$ by rescaling $\left.\zeta\right|_{\tilde{K}}$ in the next way. There is a homeomorphism $\kappa: \tilde{K} \rightarrow R_{1}$ of the form: $\left(x_{1}, x_{2}, x_{3}\right) \mapsto\left(x_{1}, x_{2}, \rho\left(x_{3}\right)\right)$ where $\rho:\left[0, z_{0}\right] \rightarrow\left[z_{1}, z_{0}\right]$ is any increasing continuous function. Then, we define $\left.\zeta\right|_{R_{1}}=\left.\kappa \xi\right|_{\tilde{K}} \kappa^{-1}$. Observe that $\zeta$ equals $\xi$ on $U^{\prime \prime}\left(z_{0}\right)$ and coincide with the identity on $U^{\prime \prime}\left(z_{1}\right)$. As a consequence, the complement part of the statement follows directly. Indeed, if $B$ lies in $K$ and $\left.\xi\right|_{\tilde{B}}=I d$ then its conjugate by $\kappa$ is the identity on $\tilde{B} \cap R_{1}$.

We continue by defining $\zeta$ on the other horizontal annuli from the previous list. As required, $\zeta$ is the identity when this annulus lies in $\mathcal{H}$. For the others, that is, $U^{\prime}\left(z_{0}\right)$ and $U\left(z_{j}\right), j=$ $0,2, \ldots, m$, Lemma 5.4 is applicable as it is explained right below.

Each of these annuli is bounded by two curves; one of the two lies in the frontier of $\mathcal{T}$ on which $\zeta$ has to coincide with $\xi$ and is mapped in the respective plane $\Pi\left(z_{j}\right)$-according to (iii); and the other lies in $\mathcal{H}$ where $\zeta$ has to coincide with $\left.I d\right|_{\mathcal{H}}$. In order to satisfy the equivariance property 3), we choose

$$
\left.(*) \quad \zeta\right|_{U\left(z_{m+1}\right)}=\left.\mathcal{E} \zeta\right|_{U^{\prime}\left(z_{0}\right)} \mathcal{E}^{-1} .
$$

Moreover, due to the $\beta$-condition, Lemma 5.4 holds and yields $\zeta$ on each of the listed horizontal annuli.

We continue by defining $\zeta$ on the vertical annuli in the above splitting of $\mathcal{V}$. When such an annulus lies in $\partial H$ (resp. $\partial \mathcal{T}$ ), we must take $\zeta=I d$ (resp. $\zeta=\xi$ ) over there. The last two annuli are $R_{0} \cap R_{1}$ and $R_{1} \cap Q_{0}$ on which $\zeta$ is already defined by conjugating by $\kappa$. Notice that the $\beta$-condition holds for these two annuli because conjugating by $\kappa$ preserves the $\beta$-condition.

Let us look more precisely to $\partial Q_{0}$. It is made of the following: two horizontal annuli $U\left(z_{0}\right)$ and $U\left(z_{2}\right)$, and three vertical ones $R_{1} \cap Q_{0}, \tilde{c}^{1} \cap E_{1}$ (lying in $\mathcal{H}$ ) and $\tilde{c} \cap\left(E_{0} \cup E_{1}\right)$. The $\beta$-condition holds for each of these latter annuli.

For finishing the proof, it remains to extend the $\zeta$ which we have defined right above on the tori $\partial R_{0}, \partial Q_{0}$ and $\partial Q_{j}, j=2, \ldots, m$ to embeddings of the solid tori $R_{0}, Q_{0}, Q_{2}, \ldots, Q_{m}$ with values in $E_{0}, E_{0} \cup E_{1}, E_{2}, \ldots, E_{m}$ respectively. The boundary data consists of annuli where $\zeta$ fulfills the $\beta$-condition. Therefore, the assumptions of Proposition 5.5 are fulfilled; then the conclusion holds true and yields the desired extention of $\zeta$ to the listed solid tori.

According to $(*)$, we can extend the $\zeta$ which is built above on a fundamental domain to $\mathcal{T} \backslash \mathcal{H}$ equivariantly. Since this extension coincides with the identity on $\mathcal{H}$, it extends by $\left.I d\right|_{O x_{1} x_{2}}$. This is a continuous extension because any point of the plane $O x_{1} x_{2}$ adheres only to $\mathcal{H} \backslash O x_{1} x_{2}$ when considering the closure of $\mathcal{T} \backslash O x_{1} x_{2}$. 
Proposition 5.2 Let $X$ be a compact topological space, $0<\tau_{1}<\tau_{2}<1$ and $\hat{\xi}: X \times\left[0, \tau_{2}\right] \rightarrow$ $X \times[0,1]$ be a topological embedding which is the identity on $X \times\{0\}, X \times\left[0, \tau_{1}\right] \subset \hat{\xi}\left(X \times\left[0, \tau_{2}\right]\right)$. Then

1. there is a homeomorphism $\hat{\zeta}: X \times\left[0, \tau_{2}\right] \rightarrow \xi\left(X \times\left[0, \tau_{2}\right]\right)$ such that $\hat{\zeta}$ is identity on $X \times\left[0, \tau_{1}\right]$ and is $\hat{\xi}$ on $X \times\left\{\tau_{2}\right\}$.

2. if for a set $B \subset X$ the equality $\left.\hat{\xi}\right|_{B \times\left[0, \tau_{2}\right]}=\left.i d\right|_{B \times\left[0, \tau_{2}\right]}$ is true then $\left.\hat{\zeta}\right|_{B \times\left[0, \tau_{2}\right]}=\left.i d\right|_{B \times\left[0, \tau_{2}\right]}$.

Proof: Let us choose $l \in\left(\tau_{1}, \tau_{2}\right)$ such that $X \times[0, l] \subset \hat{\xi}\left(X \times\left[0, \tau_{2}\right]\right)$. Define a homeomorphism $\kappa:\left[\tau_{1}, 1\right] \rightarrow[0,1]$ by the formula

$$
\kappa(t)=\left\{\begin{array}{l}
\left(x, \frac{l\left(t-\tau_{1}\right)}{l-\tau_{1}}\right), t \in\left[\tau_{1}, l\right] \\
(x, t), t \in[l, 1] .
\end{array}\right.
$$

Let $\mathcal{K}(x, t)=(x, \kappa(t))$ on $X \times\left[\tau_{1}, 1\right]$. Then the required homeomorphism can be defined by the formula

$$
\hat{\zeta}(x, t)=\left\{\begin{array}{l}
(x, t), t \in\left[0, \tau_{1}\right] \\
\left.\mathcal{K}^{-1} \xi(\mathcal{K}((x, s)))\right), s \in\left[\tau_{1}, \tau_{2}\right]
\end{array}\right.
$$

Property 2 automatically follows from this formula.

$\diamond$

We now collect some facts of geometric topology in dimension 2 and 3 on which the proof of Proposition 5.1 is based. We begin with the Schönflies Theorem (see Theorem 10.4 in [17]).

Proposition 5.3 Every topological embedding of $\mathbb{S}^{1}$ into $\mathbb{R}^{2}$ is the restriction of a global homeomorphism of $\mathbb{R}^{2}$ which is the identity map outside some compact set of the plane.

One can derive the Annulus Theorem in dimension 2; we state and prove it in the only case which we use. The coordinates of $\mathbb{R}^{2}$ are $\left(x_{1}, x_{2}\right)$. The unit closed disc in $\mathbb{R}^{2}$ is denoted by $\mathbb{D}^{2}$; its boundary is $\mathbb{S}^{1}$. The annulus $2 \mathbb{D}^{2} \backslash \operatorname{int}\left(\mathbb{D}^{2}\right)$ is denoted by $\mathbb{A}$. Let $\mathbb{I}$ denote the arc $\left\{1 \leq x_{1} \leq 2, x_{2}=0\right\}$.

Lemma 5.4 Let $g: 2 \mathbb{S}^{1} \cup \mathbb{I} \rightarrow \mathbb{R}^{2} \backslash(0,0)$ be a topological embedding which surrounds the origin in the direct sense and has the next properties: $g(\mathbb{I}) \subset\left\{x_{1}>0\right\}$, the image $g\left(2 \mathbb{S}^{1}\right)$ avoids the circle $C$ of radius $\frac{3}{2}$ and $g(1,0)$ lies inside $\frac{3}{2} \mathbb{D}^{2}$. Then $\left.g\right|_{2 \mathbb{D}^{2}}$ extends to an embedding $G: \mathbb{A} \rightarrow \mathbb{R}^{2} \backslash \operatorname{int}\left(\mathbb{D}^{2}\right)$ which coincides with the identity on $\mathbb{S}^{1}$ and maps $\mathbb{I}$ into $\left\{x_{1}>0\right\}$.

Proof: Let $p$ be the last point on $g(\mathbb{I})$ starting from $g(1,0)$ which belongs to $C$. Let $q$ be its inverse image in $\mathbb{I}$. Define $G$ on the segment $[(1,0), q]$ as the affine map whose image is $[(1,0), p]$ and take $G$ coinciding with $g$ on $[q,(2,0)]$. The image $G(\mathbb{I})$ is a simple arc in $\left\{x_{1}>0\right\}$. Because, any simple arc is tame in the plane, this definition of $G$ on $\mathbb{I}$ and the values which are imposed on the two circles $\mathbb{S}$ and $2 \mathbb{S}^{1}$ extends to a neighborhood $N$ of $\mathbb{S}^{1} \cup \mathbb{I} \cup 2 \mathbb{S}^{1}$ in $\mathbb{R}^{2}$. By taking one boundary component of $N$ one derives a parametrized simple curve $C^{\prime}$ in $\mathbb{R}^{2} \backslash \mathbb{D}^{2}$ which does not surround the origin. Therefore, by the Schönflies Theorem, $C^{\prime}$ bounds a disc $D$ in $\mathbb{R}^{2} \backslash \mathbb{D}^{2}$ 
and the parametrization of $C^{\prime}$ extends to a parametrization of $D$. This yields the complete definition of $G$.

We are now going to apply famous theorems of geometric topology in dimension 3 to a concrete situation emanating from the problem we are facing in Proposition 5.1. The setting is the following. We look at the 3 -space

$$
Y=\mathbb{A} \times[0,1]=\left\{\left(x_{1}, x_{2}, x_{3}\right) \in \mathbb{R}^{3} \mid 1 \leq x_{1}^{2}+x_{2}^{2} \leq 4,0 \leq x_{3} \leq 1\right\} .
$$

Denote $Q_{0}$ the solid torus in $Y$ limited by the next two annuli:

- the vertical annulus $A_{v}:=\left\{x_{1}^{2}+x_{2}^{2}=1,0 \leq x_{3} \leq 1\right\}$;

- the standard curved annulus $A_{0}:=\left\{x_{1}^{2}+x_{2}^{2}+\left(x_{3}-\frac{1}{2}\right)^{2}=\frac{5}{4}\right\} \cap Y$.

Observe that $A_{0}$ is contained in $Y$ and contains the two horizontal circles forming $\partial A_{v}$.

Proposition 5.5 Let $g: A_{0} \rightarrow Y$ a bi-collared (meaning that $g$ extends to an embedding $A_{0} \times(-\varepsilon,+\varepsilon)$ for some $\left.\varepsilon>0\right)$ topological embedding. It is assumed that $g$ coincides with the identity on $\partial A_{0}$ and maps the arc $\Gamma_{0}:=A_{0} \cap\left\{x_{1}>0, x_{2}=0\right\}$ to an arc $\Gamma$ in $Y \cap\left\{x_{1}>0\right\}$. Then, $g$ extends to an embedding $G: Q_{0} \rightarrow Y$ which coincides with the identity on $A_{v}$.

Proof: The image $A:=g\left(A_{0}\right)$ separates $Y$ in two components $X$ and $X^{*}$. Since $A$ is bicollared, these two domains of $Y$ are topological 3-manifolds. Therefore, we are allowed to apply E. Moise's Theorem (See [17, Chap.23 \& Theorem 35.3] for existence and [17, 36.2] for uniqueness), including the Hauptvermutung in dimension 3:

Every 3-manifold has a unique PL-structure, up to an arbitrarily small topological isotopy. Moreover, its topological boundary is a PL-submanifold.

As a consequence, $X$ and $X^{*}$ have $P L$-structures which agree on their intersection $A$. By uniqueness applied for $X \cup X^{*}$, the $P L$-structure on the union is the standard one after some $C^{0}$-small ambient isotopy. Denote by $P$ the planar surface $\left\{x_{2}=0, x_{1}>0\right\} \cap Y$. After a new $C^{0}$-small ambient isotopy in $Y$, we may assume that $A$ and $P$ are in general position. In what follows, we borrow the idea of proof from [14, Theorem 3.1 $]^{3}$.

Since $P$ intersects each connected component of $\partial A$ in one point only, we are sure that in general position $P \cap A$ is made of finitely many simple closed curves $c_{1}, \ldots, c_{k}$ in int $A$ and one arc $\gamma$ which links the two components of $\partial A$. One of the above curves is innermost in $P$, meaning that it bounds a disc in $P$ whose interior avoids $A$; let say that $c_{1}$ is so. More precisely, $c_{1}$ bounds a disc $d$ in $P$ and a disc $\delta$ in $A$. By innermost position, $d \cup \delta$ is an embedded $P L$ 2-sphere $\sigma$. As $Y$ lies in $\mathbb{R}^{3}$, this sphere bounds a 3-ball $\Delta \subset Y$. We are going to use these data in two ways.

First, we use $\delta$ for finding an isotopy $h_{t}$ of $A$ in itself from $\left.I d\right|_{A}$ to $h_{1}: A \rightarrow A$ such that $h_{1}(\Gamma) \cap \delta=\emptyset$. This is easily done as $\Gamma \cap \delta$ avoids one point $z_{\delta}$ in $\delta$ : one pushes $\Gamma \cap \delta$ along the rays of $\delta$ issued $z_{\delta}$. Notice that $h_{1}(\Gamma)$ still lies in $\left\{x_{1}>0\right\}$, but this could be no longer true for $h_{t}(\Gamma), t \neq 0,1$, when $\delta$ is not contained in $\left\{x_{1}>0\right\}$.

\footnotetext{
${ }^{3}$ In this article which we referred to, it should be meant that the $P L$-category (or smooth category) is used. Indeed, there is no general position statement in topological geometry without more specific assumption.
} 
Once, this is done, the ball $\Delta$ is used for finding an ambient isotopy of $Y$ which is supported in a neighborhood of $B$, small enough so that $h_{1}(\Gamma)$ is kept fixed, and which moves $A \cap \Delta$ to the complement of $P$. Hence, this isotopy cancels $c_{1}$ from $A \cap P$; all intersection curves contained in int $\delta$ are cancelled at the same time. By repeating isotopies similar to the two previous ones, as many times as necessary, we get an embedding $g^{\prime}: A_{0} \rightarrow Y$ which coincides with the identity on $\partial A_{0}$, still maps $\Gamma_{0}$ into $\left\{x_{1}>0\right\}$ and fulfills the following property: $A^{\prime}:=g^{\prime}\left(A_{0}\right) \cap P$ is made of one arc $\gamma^{\prime}$ only which links the two components of $g^{\prime}\left(\partial A_{0}\right)$. The annulus $A^{\prime}$ divides $Y$ into two (closed) domains $X^{\prime}$ and $X^{\prime *}$ which come from the splitting $X \cup_{A} X^{*}$ by an ambient isotopy fixing $\left\{x_{3}=0,1\right\}$ pointwise.

As $\gamma^{\prime}$ is the only intersection component of $P \cap A^{\prime}$, one knows that $\gamma^{\prime}$ divides $P$ into a disc $\mu^{\prime} \subset X^{\prime}$ (meaning a meridian disc in a solid torus) and its complement in $P$. Removing from $X^{\prime}$ a regular neighborhood of $\mu^{\prime}$ yields a $P L$ embedded 2-sphere $S$. According to the Alexander theorem [1], this sphere bounds a ball $B_{X^{\prime}}$ in $\mathbb{R}^{3}$, as $Y \subset \mathbb{R}^{3}$. It is not possible that $B_{X^{\prime}}$ contains $\mu^{\prime}$ in its interior; in the contrary, $B_{X^{\prime}}$ would get out of $X^{\prime *}$ and have a non-bounded interior. As a consequence, $X^{\prime}$ is a solid torus since it is made of a ball and a 1-handle attached. The same holds for $X$ as it is ambient isotopic to $X^{\prime}$ in $Y$.

This is not sufficient for concluding. It would be necessary to prove the same for the curve $g^{\prime}\left(\Gamma_{0}\right)$, after making it a closed curve by adding the vertical arc $\gamma_{0}^{*} \subset A_{v}$ which links the two points of $g^{\prime}\left(\Gamma_{0}\right) \cap A_{v}=\Gamma_{0} \cap A_{v}=g\left(\Gamma_{0}\right) \cap A_{v}=\Gamma_{0} \cap A_{v}=\gamma^{\prime} \cap A_{v}$. That is the place where the assumption about $g\left(\Gamma_{0}\right)$ is used.

Claim. There exists an ambient isotopy from $\left.I d\right|_{Y}$ to $k_{1}$ which is stationary on the vertical annulus $A_{v}$, which maps $A^{\prime}$ into itself and moves $g^{\prime}\left(\Gamma_{0}\right)$ to $\gamma^{\prime}$.

Proof of the Claim. Assume first that the $\operatorname{arcs} g^{\prime}\left(\Gamma_{0}\right)$ and $\gamma^{\prime}$ meet in their end points only. Consider the closed curve $\alpha$ which is made of $\gamma \cup g^{\prime}\left(\Gamma_{0}\right)$; it is contained in $\left\{x_{1}>0\right\} \cap A^{\prime}$. By construction, the homological intersection of $\alpha$ with $\gamma^{\prime}$ is zero. Therefore, $\alpha$ bounds a disc $\delta^{\prime} \subset A^{\prime}$. Notice that it could be not contained in $\left\{x_{1}>0\right\}$. The disc $\delta^{\prime}$ allows one to move $g^{\prime}\left(\Gamma_{0}\right)$ to $\gamma^{\prime}$ by an isotopy of $A^{\prime}$ into itself with the required properties.

In case where $g^{\prime}\left(\Gamma_{0}\right) \cap i n t \gamma^{\prime}$ is non-empty, in general position this intersection is made of finitely many points. Among them, choose the point $x$ which is the closest to $\gamma^{\prime} \cap\left\{x_{3}=1\right\}$ when traversing $\gamma^{\prime}$ starting from bottom. Denote by $x_{0}$ the point $\gamma^{\prime} \cap\left\{x_{3}=1\right\}$. One forms a closed curve $\tau \subset A^{\prime}$ made of two arcs in $A^{\prime}$, from $x$ to $x_{0}$ respectively in $\gamma^{\prime}$ and $g^{\prime}\left(\Gamma_{0}\right)$. For the same reason as for $\alpha$ above, the curve $\tau$ bounds a disc in $A^{\prime}$ which allows one to cancel $x$ from $g^{\prime}\left(\Gamma_{0}\right) \cap \gamma^{\prime}$ by an isotopy of $A^{\prime}$ into itself with the required properties. Iterating this process reduces us to the first case. The claim is proved. $\diamond$

Let $g^{\prime \prime}:=k_{1} g^{\prime}: A_{0} \rightarrow A^{\prime}$. As a consequence of the claim, the closed curve $g^{\prime \prime}\left(\Gamma_{0}\right) \cup \gamma_{0}^{*}$ is the boundary of the meridian disc $\mu^{\prime}$. We are going to show that $g^{\prime \prime}$ extends to an embedding $G^{\prime \prime}: Q_{0} \rightarrow X^{\prime}$ which coincides with the identity on $A_{v}$. In this aim, we denote by $\mu_{0}$ the meridian of $Q_{0}$ defined by $\mu_{0}=Q_{0} \cap P$. For beginning with, we consider regular neighborhoods $N\left(\mu_{0}\right)$ and $N\left(\mu^{\prime}\right)$ of both meridians and we extend $\left.g^{\prime \prime}\right|_{N\left(\mu_{0}\right) \cap A_{0}}$ to a homeomorphism $G_{0}^{\prime \prime}: N\left(\mu_{0}\right) \rightarrow N\left(\mu^{\prime}\right)$ which is the identity on $N\left(\mu_{0}\right) \cap A_{v}$. 
Let $B_{Q_{0}}$ be the ball in $Q_{0}$ which is the closure of $Q_{0} \backslash N\left(\mu_{0}\right)$. The restricted map $\left.G_{0}^{\prime \prime}\right|_{\partial B_{Q_{0}}}$ glued with the restriction of $g^{\prime \prime}$ to the closure of $A_{0} \backslash N\left(\mu_{0}\right)$ yields a homeomorphism

$$
G_{1}^{\prime \prime}: \partial B_{Q_{0}} \rightarrow \partial B_{X^{\prime}}
$$

The desired $G^{\prime \prime}$ is obtained by extending $G_{1}^{\prime \prime}$ to $B_{Q_{0}}$ by the cone construction (seeing a ball as the cone on its boundary). Since $g$ and $g^{\prime \prime}$ are related one to the other by an ambient isotopy fixing $A_{v}$ pointwise, an extension of $g$ follows from an extension of $g^{\prime \prime}$.

\section{References}

[1] J. Alexander, On the subdivision of 3-spaces by polyhedron, Proc. Nat. Acad. Sci. USA 10 (1924), 6-8.

[2] A. Andronov, L. Pontryagin, Rough systems, DAN. 14 No 5 (1937), 247-250.

[3] G. de Baggis, Rough systems of two differential equations Uspehi Matem. Nauk. 10 No 4 (1955), 101-126.

[4] R. Bing, The Geometric Topology of 3-Manifolds, Colloquium Publications 40, Amer. Math. Soc., 1983.

[5] Ch. Bonatti, V. Grines, Knots as topological invariant for gradient-like diffeomorphisms of the sphere $S^{3}$, Journal of Dynamical and Control Systems 6 No 4 (2000), 579-602.

[6] Ch. Bonatti, V. Grines, V. Medvedev, E. Pecou, Three-manifolds admitting Morse-Smale diffeomorphisms without heteroclinic curves, Topology and its Applications 117 (2002), $335-344$.

[7] Ch. Bonatti, V. Grines, V. Medvedev, E. Pecou, Topological classification of gradient-like diffeomorphisms on 3-manifolds, Topology 43 (2004), 369-391.

[8] Ch. Bonatti, V. Grines, O. Pochinka, Classification of the Morse-Smale diffeomorphisms with the finite set of heteroclinic orbits on 3 -manifolds, Trudy math. inst. im. V. A. Steklova 250 (2005), 5-53.

[9] Ch. Bonatti, V. Grines, O. Pochinka, Classification of Morse-Smale diffeomorphisms with the chain of saddles on 3-manifolds, 121-147 in: Foliations 2005 World Scientific, Singapore, 2006.

[10] A. Candel, Laminations with transverse structure, Topology 38 No 1 (1999), 141-165.

[11] V. Grines, V. Medvedev, O. Pochinka, E. Zhuzhoma, Global attractors and repellers for Morse-Smale diffeomorphisms, Trudy math. inst. im. V. A. Steklova 271 (2010), 111-133. 
[12] V. Grines, O. Pochinka, Morse-Smale cascades on 3-manifolds, Russian Mathematical Surveys 68 No 1 (2013), 117-173.

[13] V. Grines, T. Medvedev, O. Pochinka, Dynamical Systems on 2- and 3-Manifolds, Springer, 2016.

[14] V. Grines, E. Zhuzhoma, V. Medvedev, New relations for Morse-Smale systems with trivially embedded one dimensional separatrices, Sbornik Math. 194 No 7 (2003), 979-1007.

[15] E. Leontovich, Some mathematical works of Gorky school of A. Andronov, 116-125 in: Proc. of the third All-Union mathematical congress V. 3, 1958.

[16] A. Mayer, Rough map circle to circle, Uch. Zap. GGU 12 (1939), 215-229.

[17] E. Moise, Geometric Topology In dimensions 2 and 3, GTM 47, Springer, 1977.

[18] J. Palis, On Morse-Smale dynamical systems, Topology 8 No 4 (1969), 385-404.

[19] J. Palis, W. de Melo, Geometrical theory of dynamical systems, Mir, Moscow, 1998, Springer, 1982, ISBN: 978-1-4612-5703-5 (Online).

[20] J. Palis, S. Smale, Structural stability Theorems, Proceedings of the Institute on Global Analysis, V. 14, 223-231, Amer. Math. Soc., 1970.

[21] M. Peixoto, On structural stability, Ann. of Math. 69 No 1 (1959), 199-222.

[22] M. Peixoto, Structural stability on two-dimensional manifolds, Topology 1 No 2 (1962), 101-120.

[23] M. Peixoto, Structural stability on two-dimensional manifolds (a further remarks), Topology 2 No 2 (1963), 179-180.

[24] S. Smale, Differentiable dynamical systems Bull. Amer. Math. Soc. 73. No 6. (1967), 747817.

address: Laboratoire de Topologie, UMR 5584 du CNRS, Dijon, France email: bonatti@u-bourgogne.fr

address: National Research University Higher School of Economics, 603005, Russia, Nizhny Novgorod, B. Pecherskaya, 25

email: vgrines@yandex.ru

address: Université de Nantes, LMJL, UMR 6629 du CNRS, 44322 Nantes, France email: francois.laudenbach@univ-nantes.fr 
address: National Research University Higher School of Economics, 603005, Russia, Nizhny Novgorod, B. Pecherskaya, 25

email: olga-pochinka@yandex.ru 